\title{
UCID- 18489
}

A SUREMRY AND REVIEW OF HYYROGEN TIEORETICAL

EQUATION-OF-STATE MODEI.S AT

LALRENCE LJVIERMORE LABORATORY

H. C. Craboske, Jr.

k. L. Wong

Eebruary 1, 1980

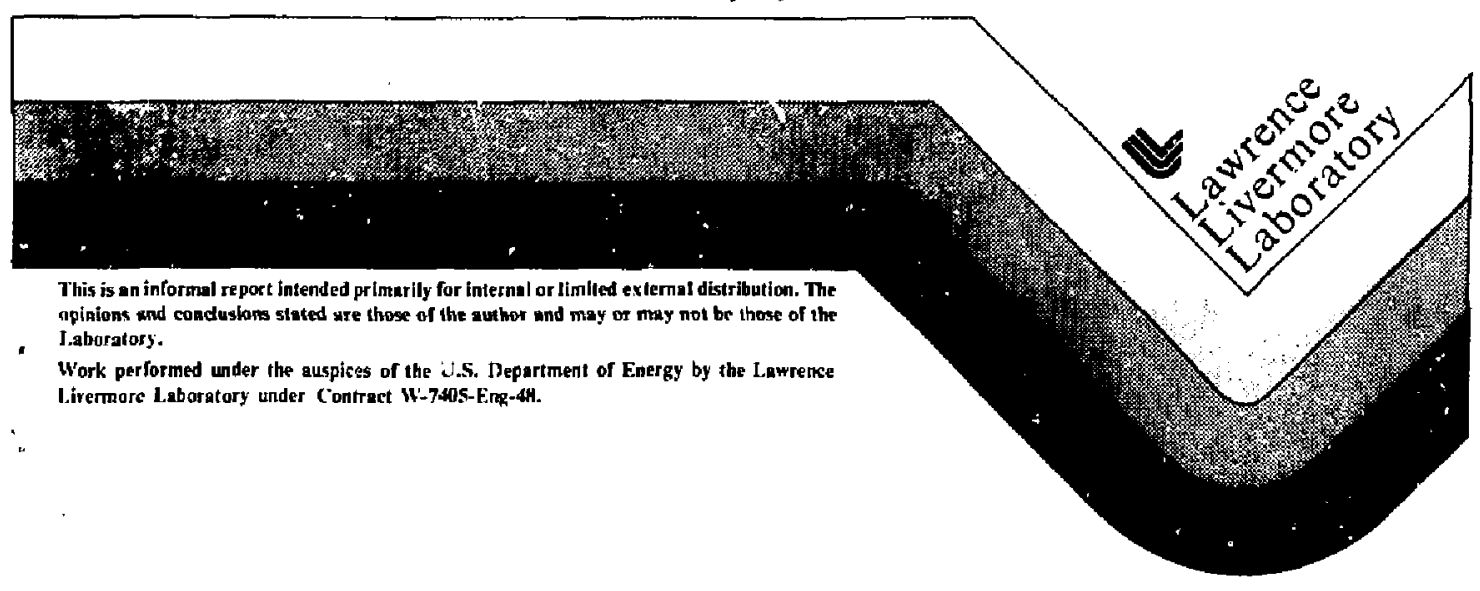

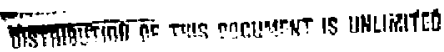


CotJuATs
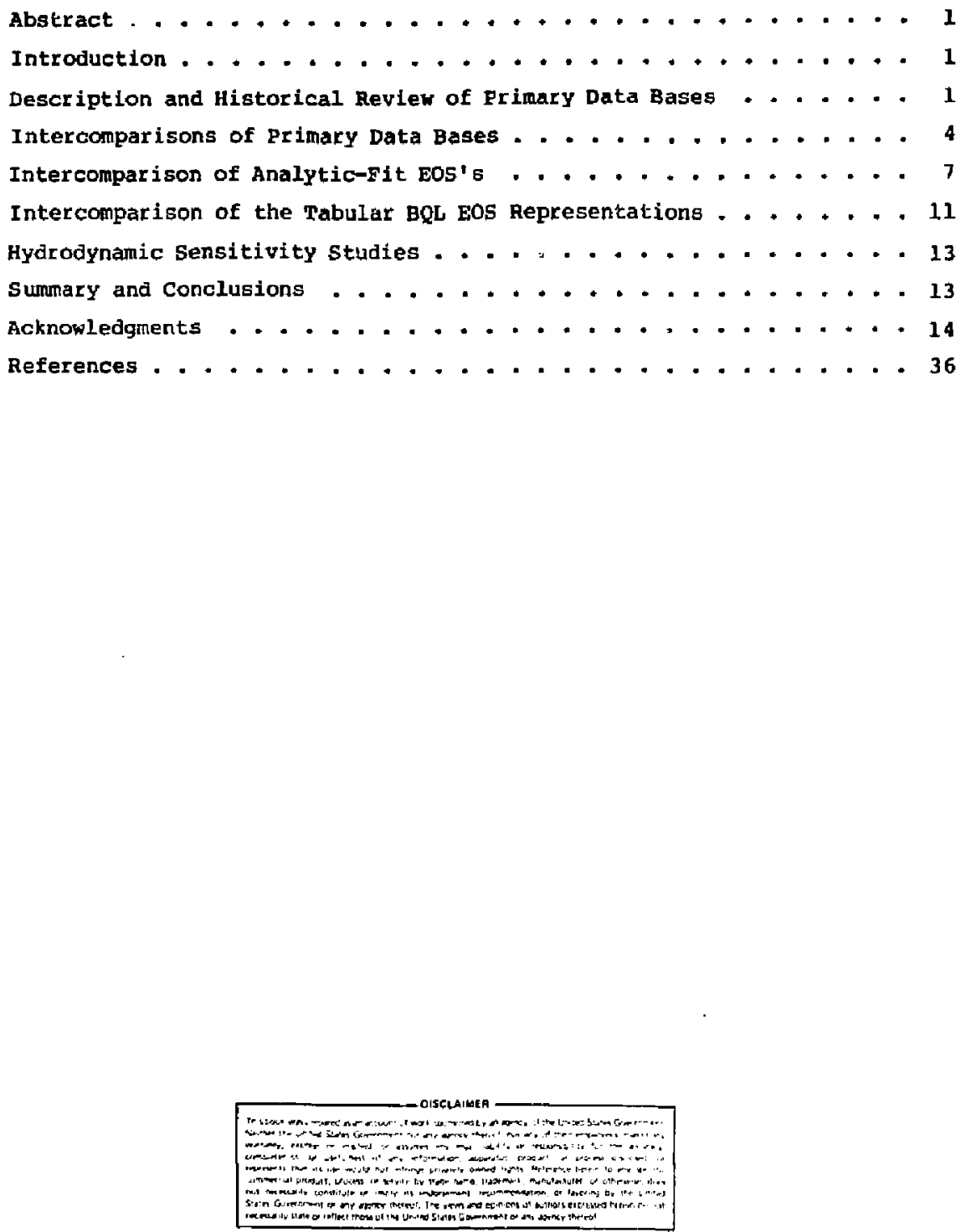


\section{A SUMAARY AND REVIEW OE HYDROGEN}

THEORETICAL EOUATION-OF-STATE MODELS

AT LAWREACE LIVERHORE LABORATORY

\section{ABSTRACT}

We present a review and analysis of the hydrogen equations of state (EOS) at use at Lawrence Livermore Laboratory (LLL). Careful comparisons to experimental data and among EOS's are performed and evaluated. We find that the new LLL hydrogen EOS (EOS95) and its representations produce the best agreement with experimental data and hydrodynamic simulation studies.

\section{INTRODUCTION}

Due to its functional importance and fundamental primacy, the physics of hydrogen * has been studied for more than 20 years at LLL. During this period there has been continuous evolution of our ability to model the high pressure thermodynamics of hydrogen. The bistery of this evolution is complicated and say be obscure in details. In this report we attempt to present a complete review of the studies of hydrogen from 1972 through 1979 , including critical evaluations and plots showing a large set of comparisons.

DESCRIPTION AND HISTORICAL REVIEW OF PRIMARY DATA BASES

Table I presents the history and evolution of the hyarogen EOS. Tabulated are (1) the primary data base (PDB) nane representing the full primary set of numericaJ. EOS data; (2) the LLL's H-Division EOs Reference File (HERF) number where references to the physics codes and construction methods used in generating the PDB can be found; (3) the analytic fit EOs (AFEos) name given to the analytic representation of the RDB; (4) the biquadratic Lagrange tabular EOS (BQLEOS) number referring to the BQL representation generated from the PDB; (5) the EOS name associated with the giant planet studies (GPEOS):

\footnotetext{
*Far simplicity throughout this report, the terins hydrogen or $\mathrm{H}_{2}$ will be used to describe-work carried out on hydrogen or deuterium.
} 
(6) a brief descriptor of the EOS; and (7) an index to a brief discussion of the origin of the PDB. The key to the origin index in riable 1 follows.

1. The original LIL hydrogen EOS (1960 to 1962), was primarily the work of Gilda Harris (circa 1962). Constructed from the low density CONARI code of Harris and Trulio with an interpolation region to join onto Cowan TED at high pressures.

2a. A composite modern LLL EOS was constructed in 1972. This work was reported in UCID-16095 $5^{1}$ and was compared in detail to k042. This PDB forms a partial basis for two later LLI EOS'S (EOS66 and EOS95). The AFEOS KO49 was a very difficult, restricted range fit in $p, E$ spaze.

2b. A substantially modified EOS from EOS6/72, is fully documented in Astrophys. $J$. 199, 255 (1975).2 Taking partial advantage of LASL and LLL hydrogen shouk wave experiments, the EOS was generated in 1973, with slight modifications made in 1974. This EOS was used in work published on giant planet evolution and was not used in F,LL hydrocode libraries.

3. Original $\mathrm{H}_{2}$ EOS from Los Alamos Scientific Laboratory (LASL) was generated by G. Kerley in 1972 and documented in Phys. Earth Plau.. Int. ${ }^{3}$ The AFEOS 37 is a restricted range fit.

4. A revision to the Kerley PDB (KNEW) just cited was made in 1973 and was received in 1975 at LLL. It is curtently the LASL standard hydrogen EOS. A restricted range fic, AFEs $55 \mathrm{~A}$, was also generated.

5. The modern analytic fit to kNEw. This fit is valid over a wide range. The fit was constrained to reproduce the PDB, but was not required to reproduce the experimental data.

6. A rectangularized modified grid extension of Eos6/72 was required for generating a BQL tabular EOS. Thermodynamic.consistency (TDC) modifications were also made. This was the LIL standard $\mathrm{H}_{2}$ PDB hefore 1978. It was used in unpublished 1977-78 giant planet studies, but was found less than satisfactory (led to cool interiors). The AFEOS KOl09 was constrained to reproduce the shock data and produce a wide range (full $\rho, E$ coverage) fit.

\footnotetext{
"The CONARI code was the predecessor to BENST, which then became FMIN.
} 
7. A revised and extended modification of EOSREV2 which has been described in detail in two informal memos (refer to $\mathrm{H}$. Graboske for aetails) and a paper. 4 This is now the standard LLL $\mathrm{H}_{2}$ EOS. The AFEOS KC95A was forcibly normalized to fit all experimental data and covers the full p.E range. The following intercomparlsoms of $\mathrm{PDB} ' \mathrm{~s}, \not F^{\prime} \mathrm{s}$, and tabular EOS's are primarily aimed at a threefold comparison between EOs66, KNEw, and E0S95, the major ULL and LASL standards. A very detailed eariier comparison of the first and second PDB's has already been dor'mented, and since they have both been superseded by the three more recent standards, we will not consider them here.

TABLE 1. History and description of hydrogen EOS's.

\begin{tabular}{|c|c|c|c|c|c|c|}
\hline PDB & HERF & GPEOS & AFEOS & BQLEOS & ORIGIN ${ }^{a}$ & DESCRIPTOR \\
\hline tost & - & -- & $\mathrm{KO} 42$ & -- & 1 & 1960 Primal $\mathrm{B}_{2}$ EOS \\
\hline $\operatorname{EOS} 6 / 72$ & 36 & & $\mathrm{KO} 49$ & 275 & $2 a$ & $\begin{array}{l}\text { First modern LLL EOS } \\
1972-73\end{array}$ \\
\hline EOSREV 1 & -- & T9JHYD & & & $2 b$ & Revision of EOS6/72 \\
\hline XFILEFIX & 37 & - & 37 & -- & 3 & $\begin{array}{l}\text { Kerley LASL } \mathrm{H}_{2} \text { EOS } \\
1972\end{array}$ \\
\hline KNEW & 55 & - & $55 A^{b}$ & -- & 4 & $\begin{array}{l}\text { Revised Kerley LASL } \\
\mathrm{H}_{2} \text { EOS } 1973\end{array}$ \\
\hline KNEW & 55 & $-\infty$ & $55 \mathrm{~B}^{\mathrm{C}}$ & -- & 5 & New AF to KNEW 1978 \\
\hline E0s66 & 66 & EHYDGPD 3 & KO109 & & 6 & $\begin{array}{l}\text { Extencied, modified } \\
\text { E0s6/72-1975 }\end{array}$ \\
\hline $\operatorname{Eos} 95$ & 95 & T9HYDJ 5 & KO95A & 277 & 7 & $\begin{array}{l}\text { Extension and } \\
\text { revision of EOSREVI- } \\
1978\end{array}$ \\
\hline
\end{tabular}

a see text for explanation and key to numbers.

benerated by the B-Division PARFAIT code.

Cenerated by the H-Division VITUS code. All stbsequent AFEos were generated with this code. 
There are nc poneral rtandards to assess the overall quality of an EOS. However, a specific set of practical standards for hydrogen has been developed in 1 Division over the years. The following criteria have been applied here:

i. Thermaynamic stability (TDS) and consigtency (TDC). (See UCIR $811^{5}$ for a quantitative analysis of these criteria.)

2. Agreement with experimental data. This is the crucial test for any theoretical model. The full set of experiments included here is as follows:

a) 300-K isotherm (Amagat tables)--idcal gas thermodynamics

b) Stewart $20-\mathrm{K}$ isothe $\mathrm{rm}$ to $20 \mathrm{kbar}^{6}$--cryogenic high pressure data.

c) Single-shock Hugoniot data--the van Thiel ${ }^{7}$ 40-kbar point, and the 210-kbar point ${ }^{B} \ldots, P, P, E$ data on the shock compressed liquid. There exists a set of independent single-shock experimental data for hydrogen from R. Dick, 9 ranging Erom 25 to 150 kbar. Foss ${ }^{10}$ has analyzed these data in connection with his study of the LLL-General Motors deuterium shock experiments and concludes that although Dick's data do not agree with van Thiel et al., the size of the uncertainties in the two sets of data are sufficiently large that a single intermolecular potential can produce a theoretical Hugoniot lying within the error bars of both works. Therefore we are referencing only the LLL-GM experimental data, and acknowledging that the IASL-Dick data would also be valid for comparisons.

d) Double-shock Hugoniot data--the van Thiel et al. ${ }^{B}$ reflected shock point at $870 \mathrm{kbar}$.

The data used in this study are 1isted in Table 2.

Although the data are sparse, and the shock data are not of high accuracy, they are all we have, and they do prove difficult to match with precision. Our comparison procedure uses interrogation codes to calculate 1sotherms (20 K and $300 \mathrm{KJ}$, principal Bugoniots (single-shock 100i), and reflected Hugoniota (double-shock loci), as well as the various T-relations (TDC indicators), and alternate forms of isentropes (TDC and TDS indicators of a aifferent type). 
TABLE 2. Hydrogen-deuterium experimental data.

\begin{tabular}{|c|c|c|c|c|}
\hline & $T, \mathbb{E V}$ & $\mathrm{mg} / \mathrm{m}^{3}$ & P, Mbar & E, Hbar $\cdot \mathrm{cm}^{3} / \mathrm{g}$ \\
\hline $\begin{array}{l}\text { Stewart pt. } \\
\mathrm{H}_{2} \text { single shook } \\
\mathrm{D}_{2} \text { single shock } \\
\mathrm{D}_{2} \text { souble ghook } \\
\text { Anagat tablus: }\end{array}$ & $\begin{array}{l}0.0043079 \\
0.094791 \\
0.38776 \\
0.603188 \\
0.02585 \\
0.02585 \\
0.02585 \\
0.02585 \\
0.02585 \\
0.02585 \\
0.02585 \\
0.02585 \\
0.02585 \\
0.02585 \\
0.02585 \\
0.02585\end{array}$ & $\begin{array}{l}0.4 \\
0.387 \\
0.584 \\
1.202 \\
1.2575 \times 10^{-3} \\
3.4132 \times 10^{-3} \\
6.1078 \times 10^{-3} \\
8.8024 \times 10^{-3} \\
1.1497 \times 10^{-2} \\
2.0479 \times 10^{-2} \\
3.0180 \times 10^{-2} \\
4.2754 \times 10^{-2} \\
5.35 .31 \times 10^{-2} \\
6.0718 \times 10^{-2} \\
7.3293 \times 10^{-2} \\
0.2225 \times 10^{-2}\end{array}$ & $\begin{array}{l}0.02 \\
0.0395 \\
0.210 \\
0.870 \\
7.7707 \times 10^{-6} \\
2.1259 \times 10^{-5} \\
3.8522 \times 10^{-5} \\
5.5936 \times 10^{-5} \\
7.3814 \times 10^{-5} \\
1.3523 \times 10^{-4} \\
2.0906 \times 10^{-4} \\
3.1395 \times 10^{-4} \\
4.1349 \times 10^{-4} \\
4.8651 \times 10^{-4} \\
6.2848 \times 10^{-4} \\
7.4259 \times 10^{-4}\end{array}$ & $\begin{array}{l}0.012 \text { (Bcaled for } D_{2} \text { ) } \\
0.0651 \text { (scoled for } D_{2} \text { ) } \\
0.8995 \\
1.375\end{array}$ \\
\hline
\end{tabular}

The key point of these comparisons is to examine how well the different FLB's exhibit TDC and TDS, how well they match the set of experimental data, and how they agree or disagree with each otier. To show the rather complex nature of the two $E O S$ surfaces $[P(T, P), E(T, O)]$, we present them in Figs. 1 and 2 for EOS95, the current LLL standard EOS. The other PDE's resemble this surface reasonably closely on the scale used here to show the full temperature and density range, from $1 \times 10^{-4} \mathrm{eV}$ to $100 \mathrm{keV}$, and from $10^{-9}$ to $3.5 \times 10^{4} \mathrm{~g} / \mathrm{cmn}^{3}$.

In Fig. 3, the shock loci computed for E0s66 are compared with the three shock data points. The princlpal Hugoniot is $20 \%$ too high at the 40-kbar point, and almost 2008 too high relative to the 210 -kbar point. These serious discrepancies demongtrate that the modifications performed to generate E0s66 from EOSREV? (which agreed much better with the experimental data) have rendered it dangeroubly inaccurate. However, the reflected shock, started from I ts correct $P_{1}(210 \mathrm{kbar}), \rho_{1}\left(0.584 \mathrm{Mg} / \mathrm{m}^{3}\right)$, E $\left(0.8995 \mathrm{Mbar} \cdot \mathrm{cm}^{3} / \mathrm{g}\right)$ values cones very cloge to the experimental point. It should be noted that if the reflected-shock calculation were initiated from the 210-kbar level on the theoretical Hugoniot (rather than the correct experimental valuel, this agreement would be lost. Also, we see fron Fig. 4, that the 20-K isotherm conly 
the 20-kbar point is plotted) and the 300-K isotherm are very accurately matched by this PDB. Overall this PDB is reasonable, but its principal Hugoniot is st: far off the experimental values that it is not a good choice to use in hydrodynamic calculations.

The KNEW PDB is used to calculate similar principal and reflected-shock loci, and is compared to the same experiments, shown in Fig. 5. The singleshock locus is in very good agreement with the shock data, being exact at 40 ktar, and $108 \mathrm{high}$ at $210 \mathrm{kbar}$. However, the 970 -kbar point lies 258 below the KNEW prediction, a serious discrepancy. The 20-K, 300-K isotherms are plotted in Fig. 6, showing excellent agreenent with the experimental data. Also present in this PDB is the two-phase (molecular-metallic) region at 2 Mbar. This data base is quite good, and except for the disagreement with the reflected shock data, is acceptable for hydrodynamic modeling.

The third PDB, EOS95, is used for a similar comparison, as shown in Figs. 7 and 8 . The agreenent with all three shock points is quite gooci, the theoretical shock loci lying about 108 high relative to the 210- and 870-kbar points. This agreement has, of course, been Forced, in that this 1978 Eos was renormalized to match the experiment as closely as passible. The reason it does not natch exactly is that the energy surface was renormalized to render it TDC (as best possible with our existing numerical interpolation codes); this second zenordalization moves the theoretical shock loci slightly away from the experimental data. However, this EOS represents the best match to the data of the three standard EOS's under discussion.

The shook loci computed from kNEw and EOS66, compared in Fig. 9, show large discrepancies on the principal hugoniot for $100 \leqslant P(k b a r) \leqslant 10,000$. The double-shock loci show a maxijum second-shock compression of about 2.4 $\mathrm{g} / \mathrm{cm}^{3}$, and the differences between these two tracks are good indicators of the differences one would see in isentropic paths over this same density range. In Fig. IG, the two shock loci are shown compared to the experimental data. In $F 10,11$, the $20-K$ and $300-R$ pressure isotherms are compared, both agreeing with the static, isothermal data. There is a cohesive loop in the KNEW surface 20-K isotherm not precent in Bos66, and a two-phase region in both KNEW isotherms at approximately 2 Mbar, also not present in E0s66.

A more significart comparison is shown in Fig. 12, showing tine shock laci calculated from KNEW and EOS95. These EOS's show excellent agreement on the principal Hugoniot to $250 \mathrm{kbar}$, but diverge dramatically from there. At 3 Mbar they predict densities on the Hugoniot that differ by a factor of 2 . 
Along the reflected shock locus, disagreement increases to 258 at 1 Mbar and again significant differences are seen near maximum compression. The double-wave structure in the EOS95 shock loci are due to pressure alssociation of the $\mathrm{H}_{2}$ molecule (the first, large peak) and then pressure ionization of the $\mathrm{H}$ atom (the second, small peak). These features are much less pronounced in the KNEW shock loci. A higher resolution view of these two Hugoniots is shown in Fig. 13, together with the shock data. Here the differences between the models are magnified, showing that although they are very close to the shock data at $210 \mathrm{kbar}$, and not too bad at $870 \mathrm{kbar}$, yet they differ dramatically just slightly higher in pressure. In Fig. 14, the theoretical isotherms are compared to the static isothermal data, both agreeing well. The cohesive loop and two-phase region in the KNEw model are absent from the EOS95 surface.

A final comparison is given between kO42 and KNEW in Fig. 15 and between KO42 and EOS95 in Fig. 16. The scale of these figures is much larger than the foregoing plots, so that the actual large discrepancies between $\mathrm{KO} 42$ and both standard PDB's are reduced. However, K042 is off at $40 \mathrm{kbar}$ by a factor of two $i$ pressure, and at $210 \mathrm{kbar}$ by 258 . In the 500-kbar to 5-Mbar range it is qualitatively different than either modern Hugoniat. From about 15 mar upwards it agrees roughly with both standard tugoniots, which is to be expected as thry are all based on $\mathrm{TF}$ theory in this regime. In sum, the k042 surface is a very bad approximation to either the experimental data, of the moaern EOS surfaces accepted as standards.

\section{INTERCOHPARISON OF ANALYTIC-FIT EOS'S}

For use in hydrodynamic simulation codes, the PDB's must be converted to either analytical fits or fast tabular interpolation forms. In this section we will review the analytic fit Eos representations. These range frcm a simple Grüneisen model, to polynomial forms, to the most complex LLL method-the 28-term ratio of polynomial form (28 ROP for brevity). This sequence describes an evolutionary trend in AFEos's that occurred over the period 1963-1978. The early simpler AF's (Grineisen and polynomial) are fast but are limited in range usually to the 1-to-10-Mbar pressure range, and to a segment of $P(E, P)$ surface lying near or between the principal flugoniot and the principal adiabat. Points that lay well away from this surface segment could be in error (relative to the PDE) by many orders of magnitude. Also, severe 
pathologies (poles, negative pressures, negative compressibilities) rould also occur over extensive regions. In the last few years, the more complex 28 RDP was designed to represent the entire $P(E, \rho)$ surface, and eliminate or greatly reduce singularities and negative $P^{\prime} s$ and $B_{T}$ 's. It could extrapolate in all directions very accurately, and was given the capability of being "laced" or strongly tied to either selected parts of the PLB surface or to a selected set of the experimental data. Various demonstrations of these capabilities will be evident as we analyze and exhibit the various hydrogen AFEOS surfaces. Our analysis of fit adequacy (by use of extensive plot comparisons) is based on earlier assessments that used the rms error of $\left(\left.\right|_{f i . t}-P_{P D B} \mid / P_{P D B}\right)$ as a key adequacy eriterion, together with a nonpathological surface in the limited $P(E, \rho)$ range between the principal Hugoniot and the principal adiabat. Due to poor analysis tools, previously accepteo fits would pass the above tests, yet be a poor match to the experimental data. They could include pathologies, substantially distort certain hydrodynamic paths, or significantly amplify existing distortions in the PDB. To ensure that these defects are detected, wo chose the set of plots that will be discussed later in this report. It should be noted that these AFros are intended for pure hydrodynamic modeling, so they represent $P$ as a function of $E$ and $p$, the temperature variable being elimirated. Also, they are least squares fits, so the $\mathrm{AF}$ surfaces are only approximations to the exact PDB surface, typical rms deviations of the pressures ( $\Delta P / P$ ) being in the range of 3 to 108 . There are times when a fit to the PDB is also forced to fit experimental data points where the PDB does not pass exactly through the experimental data. In this case there are two noncongruent surfaces Fresented to the fitting code, thereby resulting in deviations from one or the ather. Such discrepancies will be noted in comparisons to follow.

The AF'B examined in this study 1nclude; KO49, a restricted range fit to the EOSREVI PDB (or an earlier predecessor of this PDB\}, which was not forced to either PDB or experimental data; ko109, a recen's wide range AF to EOS66 and constrained to the three shock data points; AF 55A, an early AF to the KNEW PDB with a restricted range and not force fit; AF 55B, a refit of kNEW which was forced to fit the PDB in the 100-kbar to 10-Mbar range and was applicable over a wfde range; and AF 95A, a wide range fit to EOS95, forced to fit the three shock data points. These fitg are evaluated in terms of their cms fit to the underlying PDB, a given in the various HERF reports. ${ }^{11}$ The plots discussed here show how the overall $P(E, D)$ sur face is covered by an AF 
generated surface. Figure 16 shows how the restricted-range AF's depart radically from the correct EOS surface uutside their range of $f i t$, where is an accurate $P(E, \rho)$ AF surface is depicted for hydrogen.

First we examine the K049 AF surface, used from 1974-\% for certain studies. This surface (Fig. 17) is reasonable from 0.1 to $100 \mathrm{~g} / \mathrm{cm}^{3}$ and either pathological or grossly in error outside that range. In Fig. 18, all those points where $\triangle P / P$ (fit minus $P D B$ over the $P D B$ value, i.e., fractional pressure error) exceeds 208 is plotted, showing concentrations of ercor ir. three areas. The area where no errors greater than $20 \%$ are found is the range over which the fit was made. A more significant comparison is given in Fig. 19, where the principal Hugoniot and reflected shock paths are given for k049 and for E0s66. The agreement is quite good up to $20 \mathrm{Mbar}$, which covers the main area of interest. A higher resoiution plot, Fig. 20, shows the agreenent of these models with the experimental shock data. Typical of AF's \{also visible in Fig. 19) is their inability to follow structure or rapid change in the PDB surface. One unfortunate feature of K049 is that it çlves a much stiffer double-shock locus than the PDE, thus missing the 870-kbar point by 200 kbar. This AFEOS is clearly a poor choice for hydrogen, at least in the pressure density range given in Fig. 20 .

Next let ur examine the two AF's made to the LASL kNEw database. An earlier AFEOS, described in HERF 37 was a restricted range AF to an earlier Ferley $D_{2}$ EOS, but this is superseded by the fits described here. In Fig. 21, the AF 55A surface is calculated over the full Tro range of the KNEW PDB, showing its character as a restricted range fit with severe pathologies at lower densities and higher temperatures. For reasons that are not clear to us, both the LLL and LASL hydrogen data bases were rather difficult to model by good $A F^{\prime} S$. The $\triangle P / P \geq 20$ o error points are superimposed on this same surface in Fig. 22. The region of good fit is very restricted. The final comparison for this fit; Fig. 23, shows a reflectedshock path for AF 55A that lles 500 kbar below the same path calculated from the kNEW PDB at the density of the 870-kbar shock experiment. This set of curves identified AF $55 \mathrm{~A}$ as a very poor EOS representation, which distorted the surface too badly to be usable. We proceeded to carry out a higher quality fit using the vITus code, the result designated as AF 55B.

The resulting AFros surface is 1llustrated in Fig. 24, showing an excellent wide range $A F$, which will extrapolate nicely to high arm low $\rho$, and to high T. There are no evident pathologies (aJ.though sharp singularities 
can exist and not be visible in such a plot due to the point spacing). The $\Delta P / P \geq 20$ error points are concentrated along the low temperature isotherms in the range $I \leq \rho / \rho_{1 i q} \leq 100$, as is reasonable due to the strong curvature of these isotherms and the discontinuities produced by the two-phase region ( 1.5 to $\left.2 \mathrm{~g} / \mathrm{cm}^{3}\right)$. A further comparison of the principal Hugoniot and 210-kbar (initial pressure) double-shock locus, in Fig. 25, shows very good replication of the PDB paths by the AF. A bigher resolution plot, Fig. 26 , shows how good this fit js, only losing perfect registration where the Hugoniots exhibit rapid curvature. By lacing or forcing the AF tightly to the PDB surface, this AFEOS gives a hydrodyramic representation that is very close to the PDB itself. Note that by choosing to force the fit to the PDB and not to the experimental data, the double-shock locus predicted by AF 55B will lie some 400 kbar above the B70-kbar experimental point, as does the PDB locus. A final comparison, rig. 27 , is between $A F$ and $P D B$ along the $20-K$ and $300-K$ isotherms. The deviation of the $20-\mathrm{K}$ isotherm is probably due to not being included in the data set that was used to produce AF 55B. Also, we see that the fits do a good job of "smoothing" through the phase transition.

The next AFEOS to be considered is ROl09, the AF to the EOS66 PDB. The full $\rho, T$ range sir face generated by the Rolog AF is displayed in rig. 28 , showing the same wide range, excellent extrapolatory character and apparent freedom from pathology as seen in the previour fit. The same clustering of $\Delta P / P \geq 208$ errors in the $1 \leq \rho / \rho_{l i q} \leq 100$, low $T$ range is seen, again due to rapialy changing character of the pressure surface here (Fig. 29). An additional set of error points is evident, at low density and in the 1-to-10-e" range. These points are here becallse EOS66 contains a detailed treatnent of the $\mathrm{H}_{2}$ molecular dissociation (the lower irregular band, $10^{-7}$ to $10^{-2} \mathrm{~g} / \mathrm{cm}^{3}$ ) and the $\mathrm{H}$-ionization regions (the upper band), which are only treated apparently in an average form in the kNEW data base. In Fig. 30 , the principal Hugoniot and double-shock locus are given for both k0109 and E0S66. The AF was fit to EOS66 with a strong weighting toward the experimental shock points, and the result of such a procedure is evident. Where the PDB Hugoniot passes far above the 2l0-kbar point, the AF lugoniot lies quite close to it. However, the price paid for trying to force the AF to pass through two noncongruent surfaces (the PDB and the surface defined by the three shock points) shows by the cutoff of the AF principal Hugoniot at 500 kbar, indicating there were convergence problems in continuing the calculation beyond this point. This patholegy is probably related to the force-fitting 
procedure, but this does not necessarily imply that this afeos will cauge hydrodynamic problems as the codes use a von Neumann 0 to generate shock s"ates. As seen in Fig. 31, the reflected-shock locus from the AF lies 200 kbar above the experimental point, which is poor but acceptable. A final comparison of the 20-K and 300-K isctherms is given in Fig. 32 , for the K0109 and E0S66 rurfaces, again compared to the experimental data. Registration is excellent at $300 \mathrm{~K}$, and adequate for $20 \mathrm{~K}$ at $\rho<\rho_{1 \text { iq }}$. The KOlOS afEOS reftesents an actual improvement over the PDB (EOS66) From which it was generated, in that it is a ciose fit overall, and produces a much better principal hugoniot. However, the severe deficiencies of eOs66 make 10109 a less desirable choice than the newer EOS to be described.

The final hydrogen EOS, EOS95, was a further modification of the LLL sequence, attempting to improve the overall quality by forcing a match to the experimental data. The AF 95A fit to this surface is illustrated in Fig. 33. showing the same ": gh quality wide range features as seen for k0109. The quality of this fit was so good that there were very few errors greater than 1DB. The $\triangle P / P \geq 108$ error points are suptrimposed on the $A F 95 A$ surface in Fig. 34, far fewer in number than previous fits, but still lacated in the low-T, medium-dersity region and along the dissociation and ionization zones. In Fig. 35, the two standard-shock loci are plotted. The inability of these analytic fits to follow rapidy changing surfaces is more evident in this surface which has more structure and detail than any of the others. A cloaer comparison of the shock loci is given in Fig. 36, as well as the experimental data. The fit passes almost exactly through the experimental shock data, as expected, but tiłe pressure dissociation loops are displaced relative to the FDB Hugoniot. A fina? comparison is given for the 20-K and 300-K isotherms with the static data in Fig. 37. Again the agreement is good. Our final assessient is that EOS95 and its AF, 95A, are the best available hydrogen thermodynamic models available at present.

\section{INTERCOHPARISON OF THE TABULAR BQL EOS REPRESENTATIONS}

A second and completely different type of numerical representation of the EOS primary data base $1 \mathrm{~s}$ the use of some numerical interpolation algorithm chosen to fit the data, which is then reduced to a tabular array (two-dimensional, usually in $T$ and $\rho$ ) stored for pressure and internal energy in a material properties library. Two different forms used by us are 
the biquadratic Lagiange interpolation (BQL) and the Evor form, ${ }^{4}$ a bilinear interpolation in logarithmic variables, with Eos derivatives computed by a higher order method and also tabulated along with the two EOS's.

In Fig. 38, a high resolution plot of the two shock loci from BoL275 and the EOS66 primary data base are compared to the experimental shock points. The BQL EOS is unfortunately even farther away from the data than tha PDB loci, that is, the fit procedure producing the tabular form has distorted the surface slightly, but in a way that worsens its Hugoniot predictions. BoLi75 should be avoided for modeling hydrogen at pressures below 50 Mbar.

The ccinparison of the principa. Hugoniot and the double-shock locus are shown in Fig. 39, for BQL277 and the EOS95 PDB. As is evident, the agreemert is excellent, the double loop structure in both PDB loci bping closely represented by the tabular gos curves. The only deviations are in the range of 4 to 50 kbar and are unimportant. A closer comparison of these same curves is presented in Fig. 40, this time with the experimental shock data added. The BQL shock paths are as good as chose derived directly from the PDB; the 210-kbar foint lying exactly on the bQt principal Hugoniot. The maximum diccrepancies (above $50 \mathrm{kbar}$ ) between the two sets of shock loci are no more than 10 in pressure, and are usually less. A final test of the BQL 277 surface is given in Fig. 41, where the $20-K$ and $300-K$ isotherms from BQL 277 and EOS95 are compared, and the static isothermal experimental data are added. The 300-K isotherm is accurately represented, but the 20-kbar, 20-K static point is not, probably due to the truncation of the PLR data used in the actual BQL fit generation (data below room temperature are often deleted from PDB's, if present, because they are not generally relevant to hydrocode simulations). Orecall, the BQL representation of EOS95 is quite good, and is adequate to represent the ifl standard hydrogen Eos until either an improved theoretical model is completed, or better, until croproved experimental shock data are acquired.

It is worth emphasizing here how important experimental shock data have been, as they represent the extreme limit, in temperature and density, of our true knowledge of the hydrogen EOS. Clearly more shock data, of higher accuracy and in the higher pressure regime are very badly needed if we are to improve our thermodynamic models. 
Studies of tow a material's thermodyriandic properties affect its hydrocijnamic response in differont zonditions have been carpied out for a vaciaty of materials in difterent gunetries and different pressuretemperature regiturs. Two tuch etudies have been documerted fnr hydrogen, and sume data for a erird will be briefly djecussed here.

The first documented hydrogen fDS hidrodynamic analysis.is brisically a comparison of high prescute hydrodynamic tesponse between the AFExi's, KO4 and K049. The basic conclusion was that there wece substantial reasons for preferring KO49 over KO42. The second documented study, by Marino, 12 which was recent?y completed, consisted of a hydrodynamic analysis assessing the adeguacy of the analytir fits of the two standard POB's, KNEW and EOS95.

Ir adrition to these studies, G. Carlson has provided some densitytemperature loci that are of interest in applying the hydrogen EOS. A sequence of high pressure, high compression points are plotted in Fig. 42, where the given values are energy, E, and density, 0 . Fron these Erp vaiues

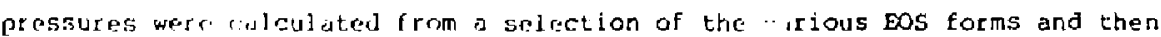
wite plotted. Also included are the three shock dala points, both the experimental $P, r$, values, and the various bos predictions for these points. We see that AF 95A does the best job of matching the experimental Hugoniot points. In addition, there are large differences (on the order of A08) between BOS's along the Carlson track. AF 55B approaches and agrees with AF $95 \mathrm{~A}$ at high compressions, where KO42 remains in poor agreement along the entire path. For high compression, high energy applicatiuns, AF $55 B$ and $A F 95 \AA$ are equivalent. However, at Iow compressions, the differences can be substantial and AF $95 \mathrm{~A}$ is probably the best representation in that regime.

SUMMARY AND CONCLUSIONS

Throughout this study, we have found EOS 95 and its representations to be the best data set for a wide range of applications, Its agreement with experiment is good and its use in hydrodynamic studies has given very satisfactory resilts. 
There is a need, however, for mora high quality experimental data in order to better define the compression regime of hjdrogen. This will not only help in constraining the representations, but can also give definition to the BOS surface where we have had little help from theoretical models.

In addition. EOS'95 does not include the molecular-metallic phase transition (as KNEW has) and this should be consigered in the next generation of the hydrogen EOS.

\section{ACXNOWLEDGMENTS}

This work represents an effort spanning the period 1972-79, and at times involved various cuntrihutors. We acknowledge their work with pleasure. The H-Division contributors included Bob 0lness, Forrest Rogers, Keith Johnson, Dick Grover. Marvin Ross, Bill Heover, and Mat van Thiel. The Laboratory's other physicists who helped included Dan Steinberg. John Morrison, Larry Marino, and Gary carlson. Outsije contributors were AI Grossman, Gerry Kerley, and Bill Hubbard. 


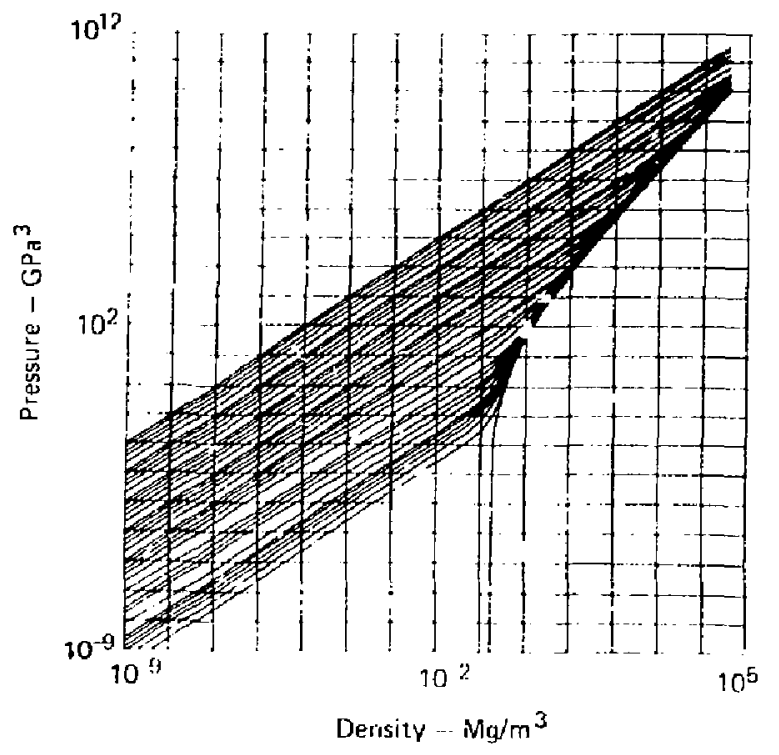

FIG. 1. Pressure EOS as a

function of $P$, with $T$ as

a parameter for EOS95 for the fi.ll $\mathrm{T}$, $\mathrm{r}$ ange.

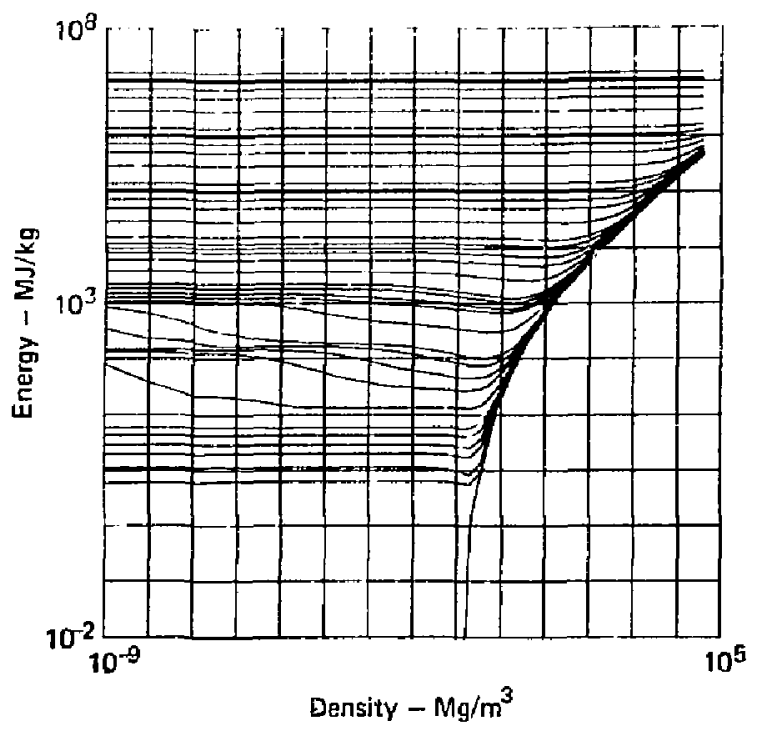

FIG. 2. Energy EOS for EOS95 for the full T,p range. 

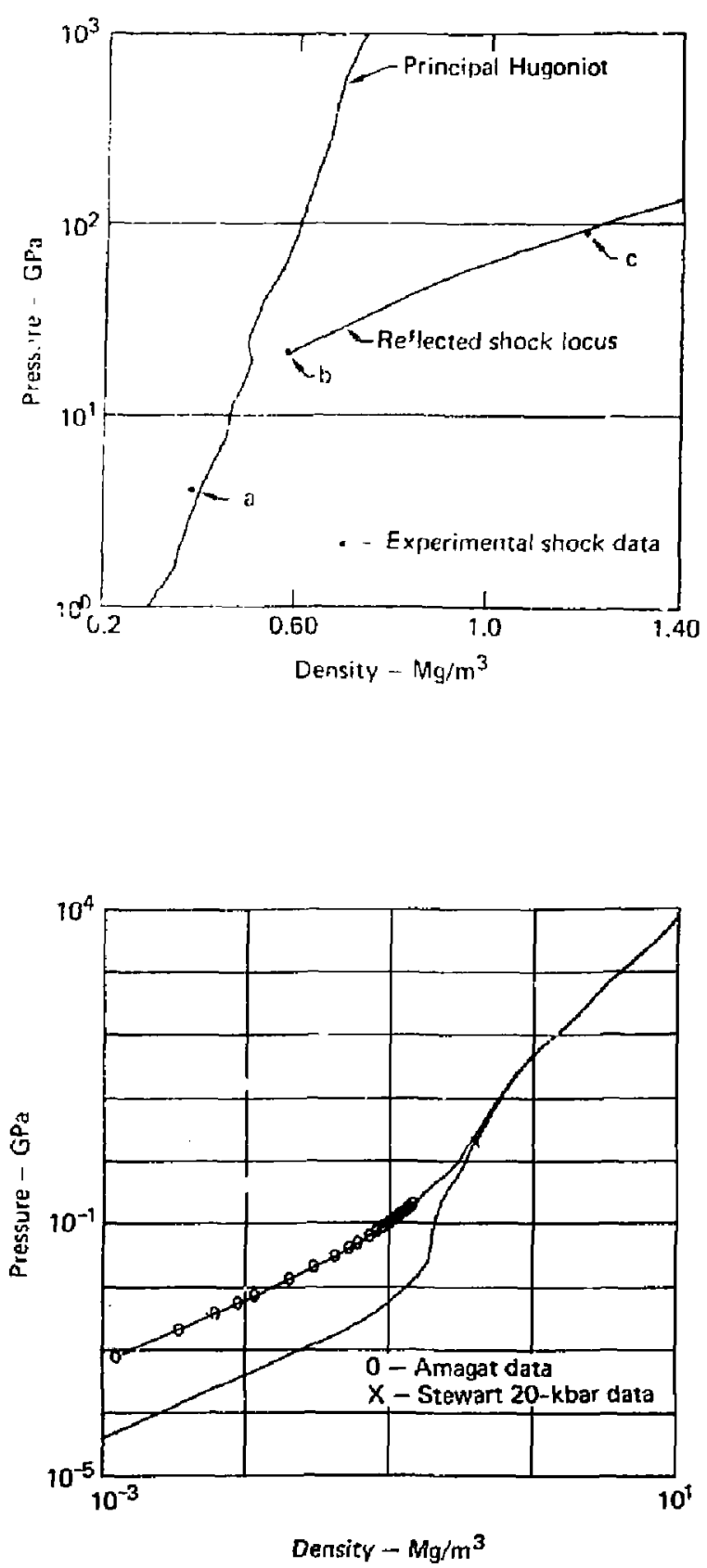

FIG. 3. Principal Hugoniot, and reflected-shock locus from the 2l0-kbar point, calculated from EOS66 PDB, compared to the (a) 40- and (b) 210-kbar single-shock experimental data points, and the (c) 870-kbar double-shock point.

FIG 4 4. The $20-k$ and $300-K$ pressure isotherms from EOS66, compared to the experimental data. 

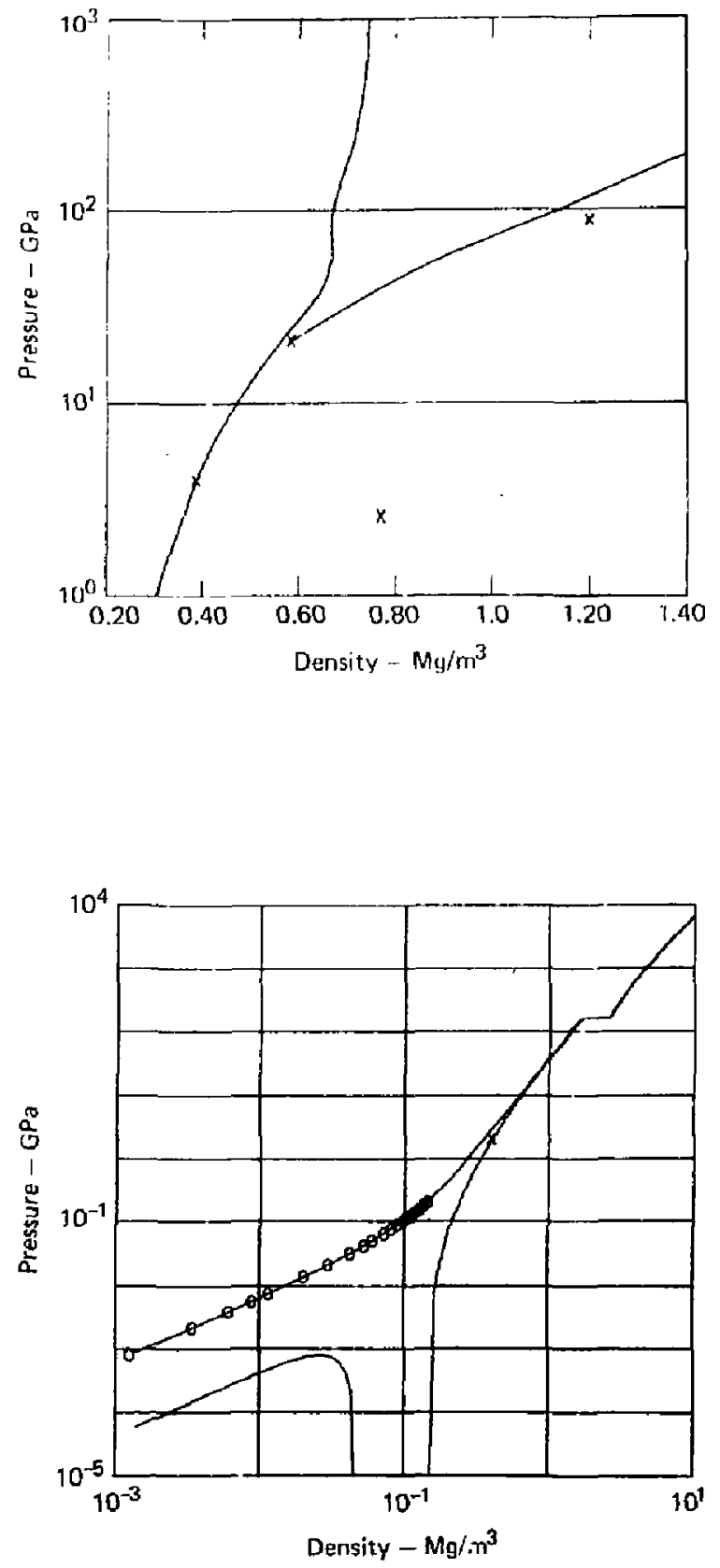

FIG. 5. Principal Hugoniot (PH) and ceflected-shock locus (RSI) from the 210 kbar point, calculated from KNEW PDB compared to the experimental data $(X)$.
FIG. 6. The KNEW PDB 20-K and 300-K isotherms, compared to the experimental data. 

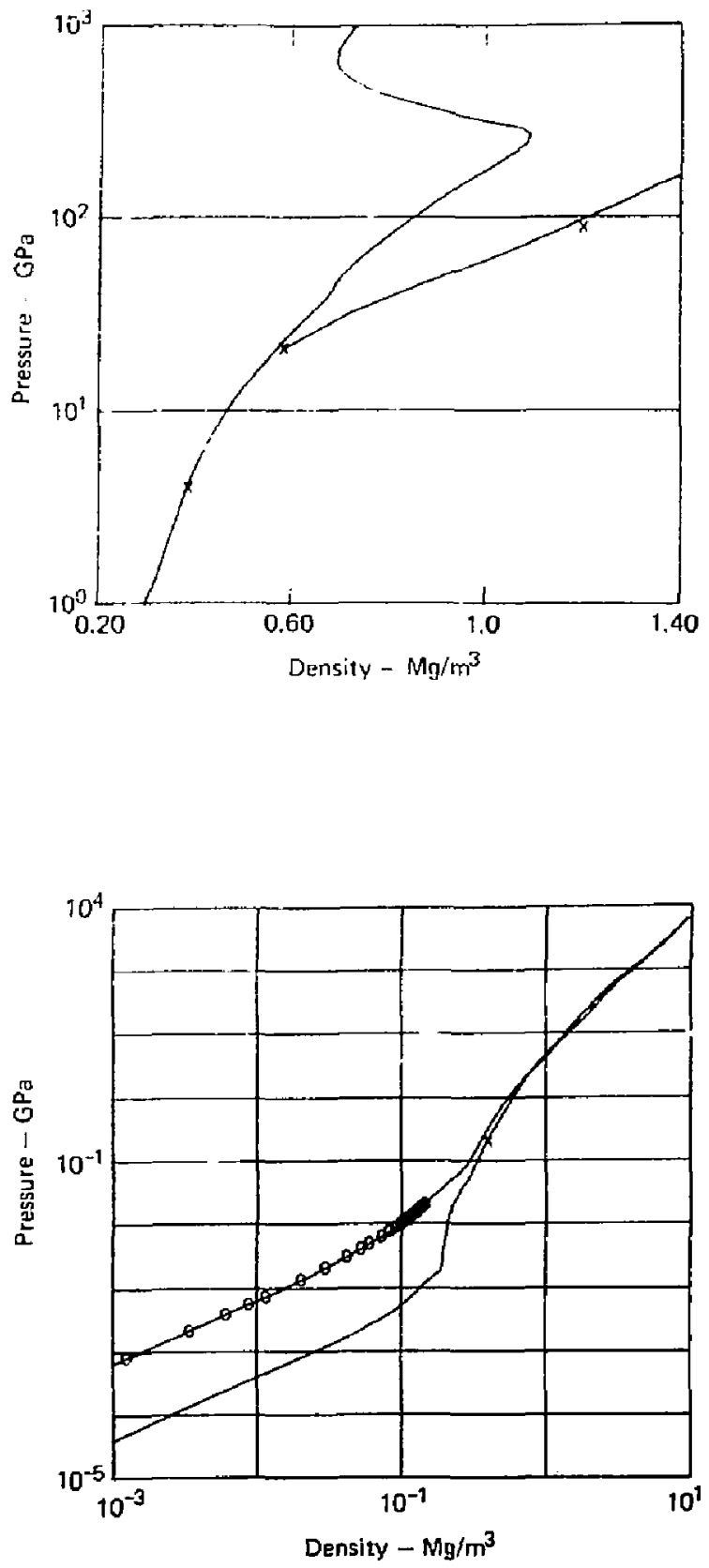

18
FIG. 7. The EOS95 PDB principal and reflected liugoniots, compared to the shock experiments.
FIG. B. The EOS95 PDB 20-K and 300-K isotherms, comwared to the experimental data. 

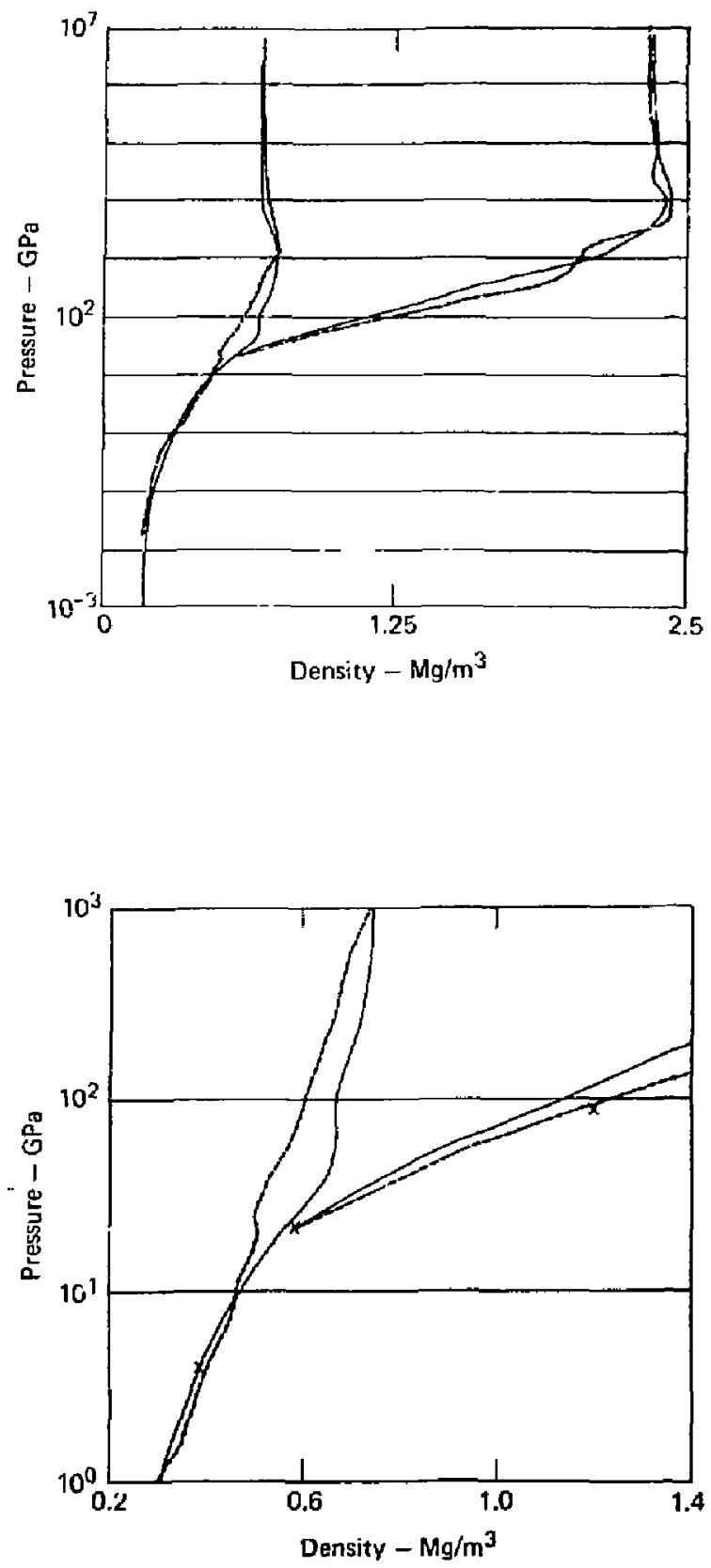

FIG. 9. Shock loci (PA and RSL) from kNEW and EOS66.
FIG. 10. Shock loci from KNEW and EOS66 compared to the experimental data. 

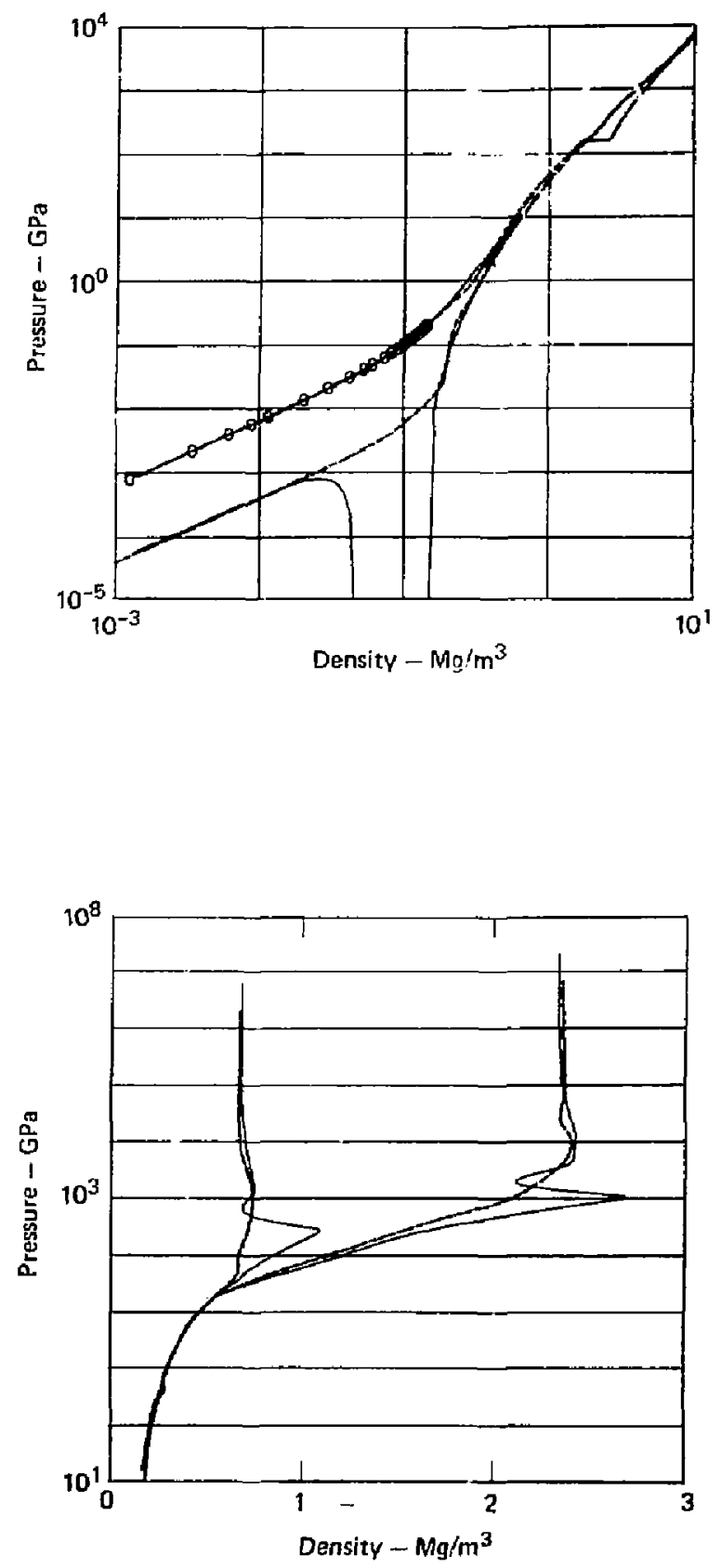

FIG. 11. The 20-K and 300-K isotherms from KNEN and EOS66 compared with static data.
FIG. 12. Shock loci from KNEW and EOS95. Note the qualitative differences in these two theoretical models that appear above 200 kbar on the $\mathrm{PH}$ and above 1 Mbar on the RSL. 

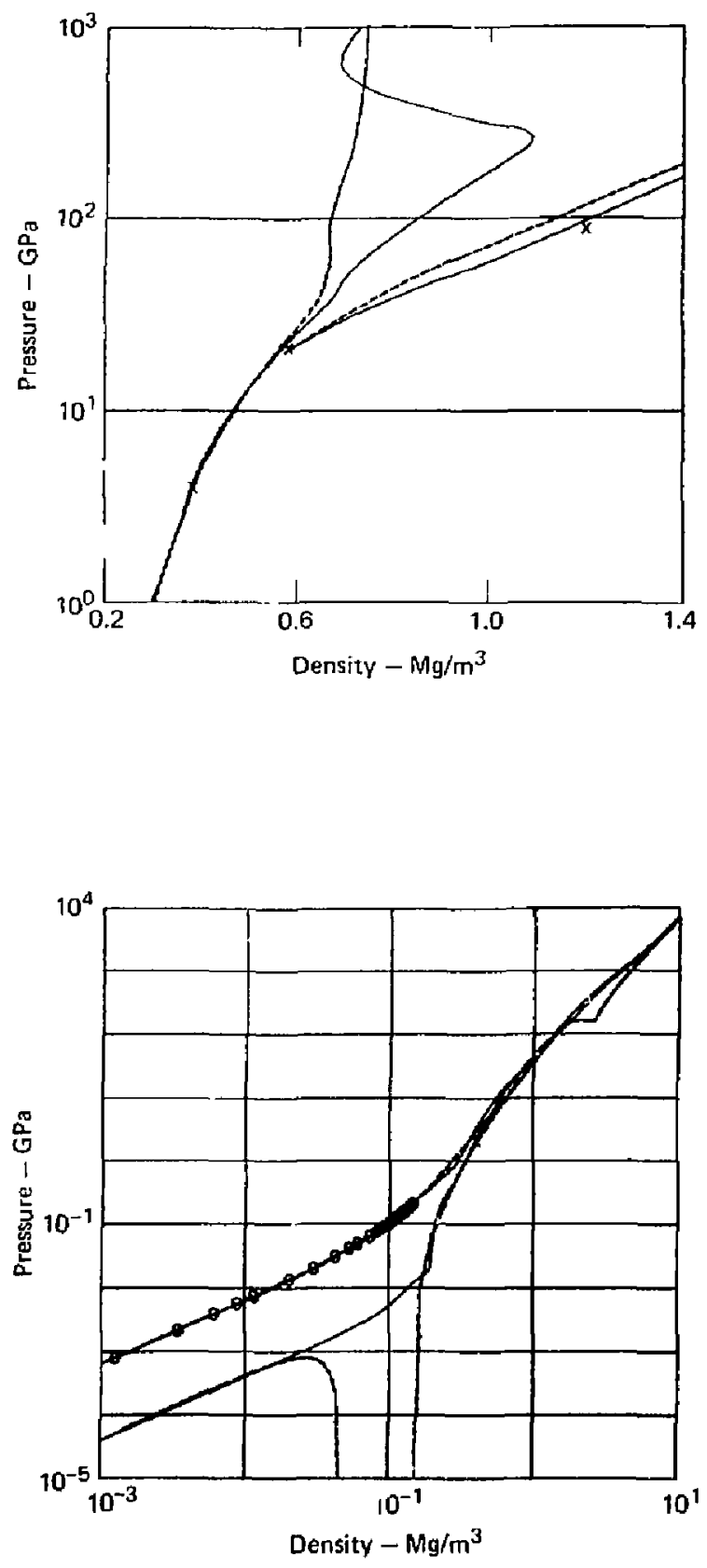

FIG. 13. Shock loci from KWEW and EOS95 compared to the experimental shock data.
FIG. 14. The 20-K and 300-K isotherms from KNEW and Eos95, compared to the static isothermal data. 

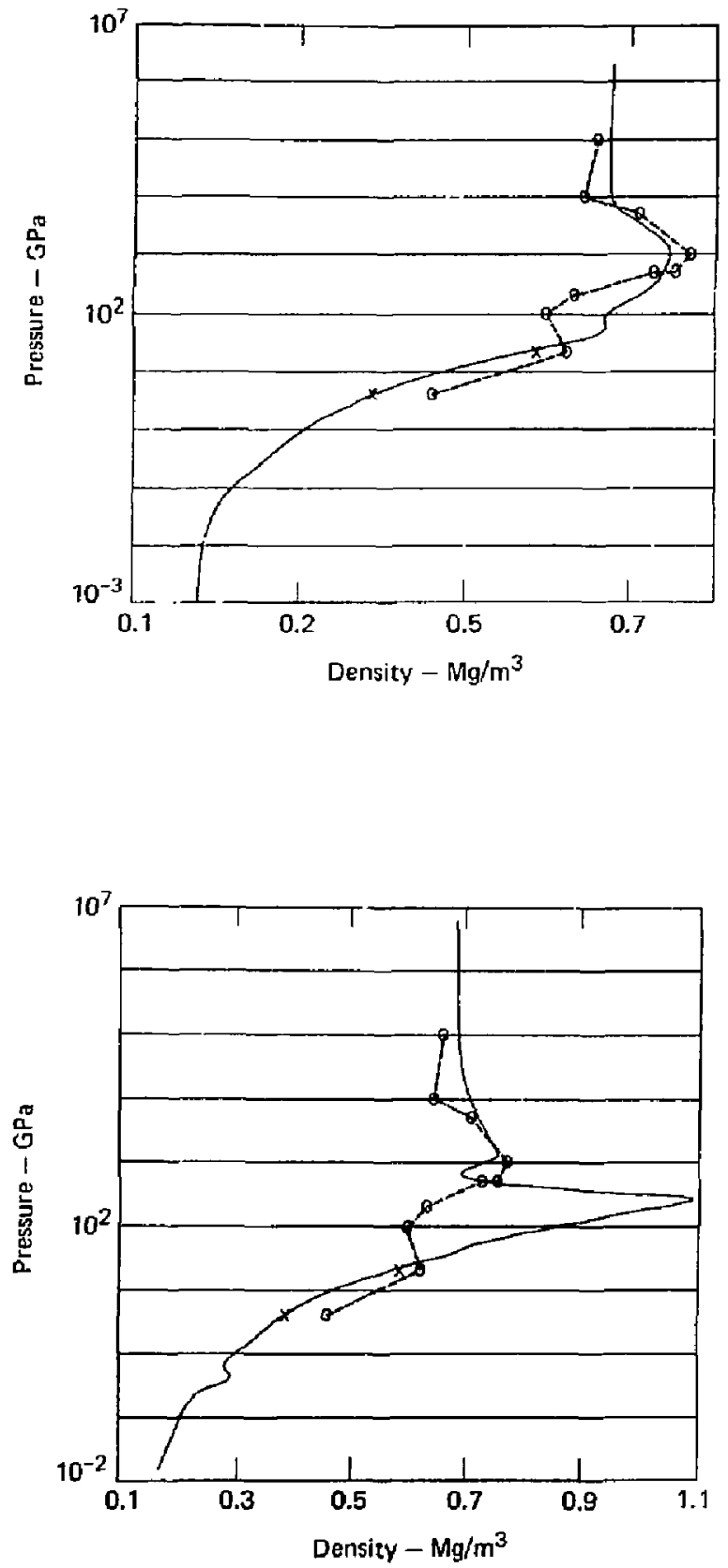

FIs. 15. Principal Hugoniot from KO42 (ccmputed by performing shock crmpressions using the KOVEC codel and KANEA.
FIG. 16. Prineipal Hugoniot from kO42 and EOS95. 

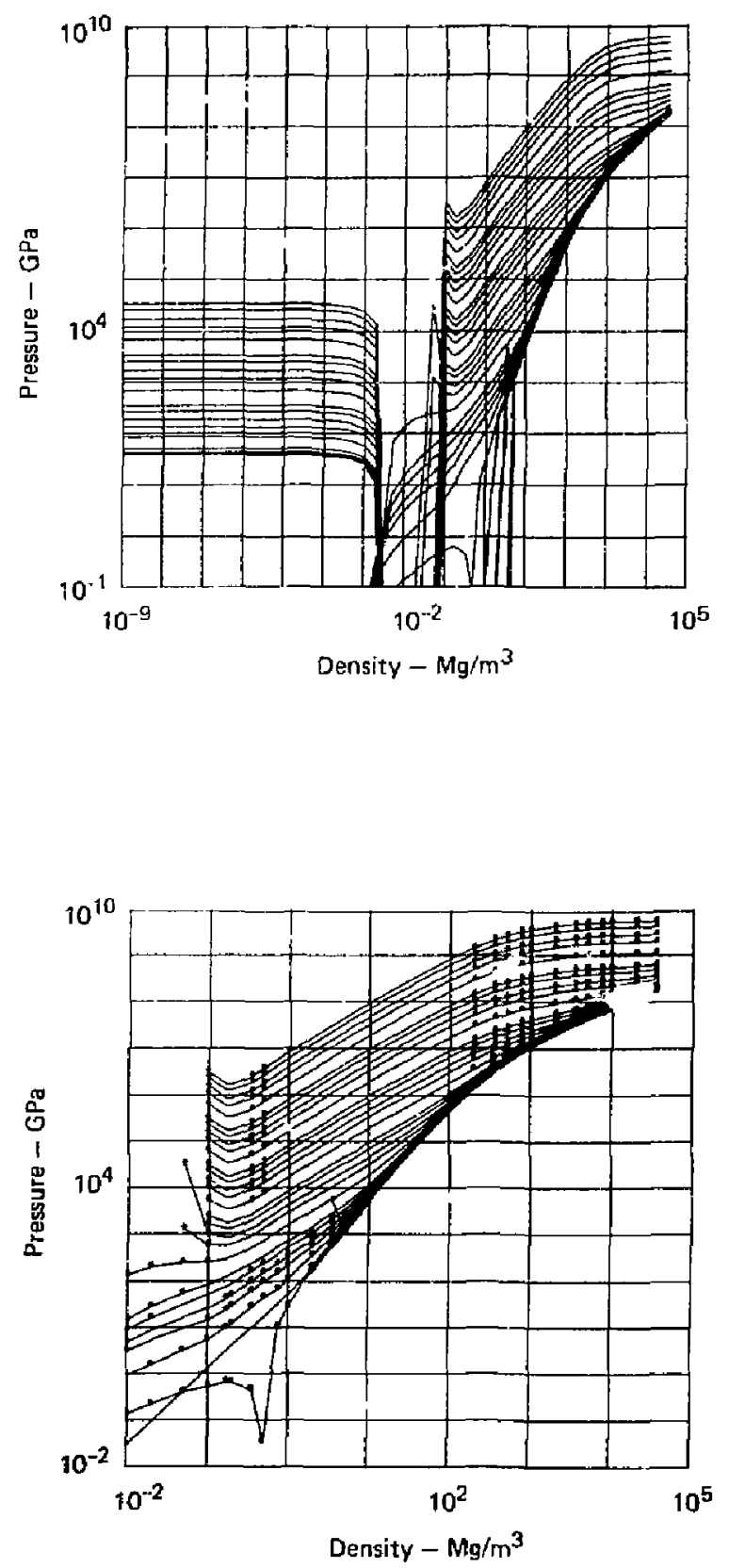

FIG. 17. KO49 AFBOS (pressure as a function of $\rho$ for constant energy as a parameter) evaluated over the $T, \rho$ range of EOS66.
FIG. 19. KO49 AFEOS $\triangle P / P$ $\geq 208$ pressure errors relative to Bos66 PDB values. 

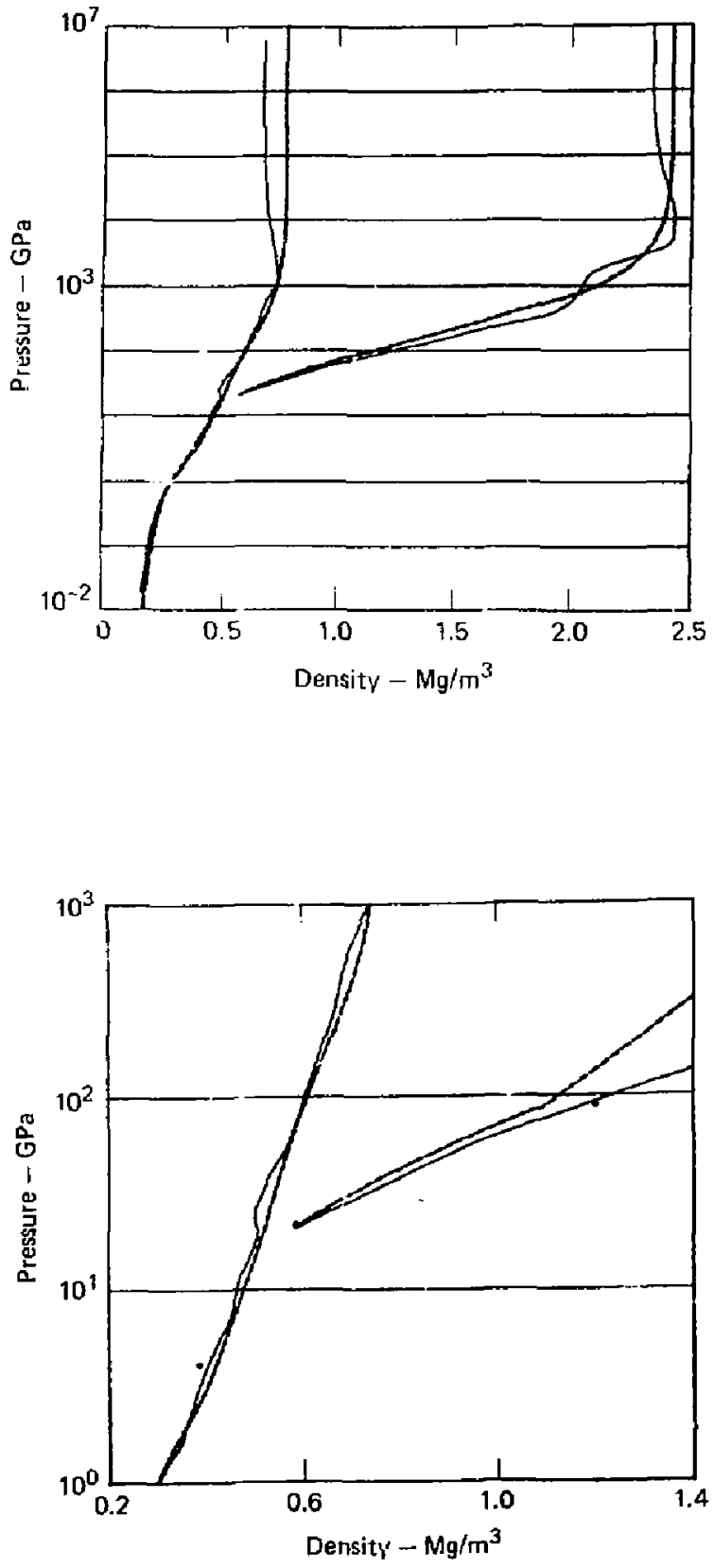

IIG, 19. KO49 principal and reflected-shock loci compared to equivalent E0s66 paths.
FIG. 20. KO49 and EOS66 shock loci compared to the shock data. 

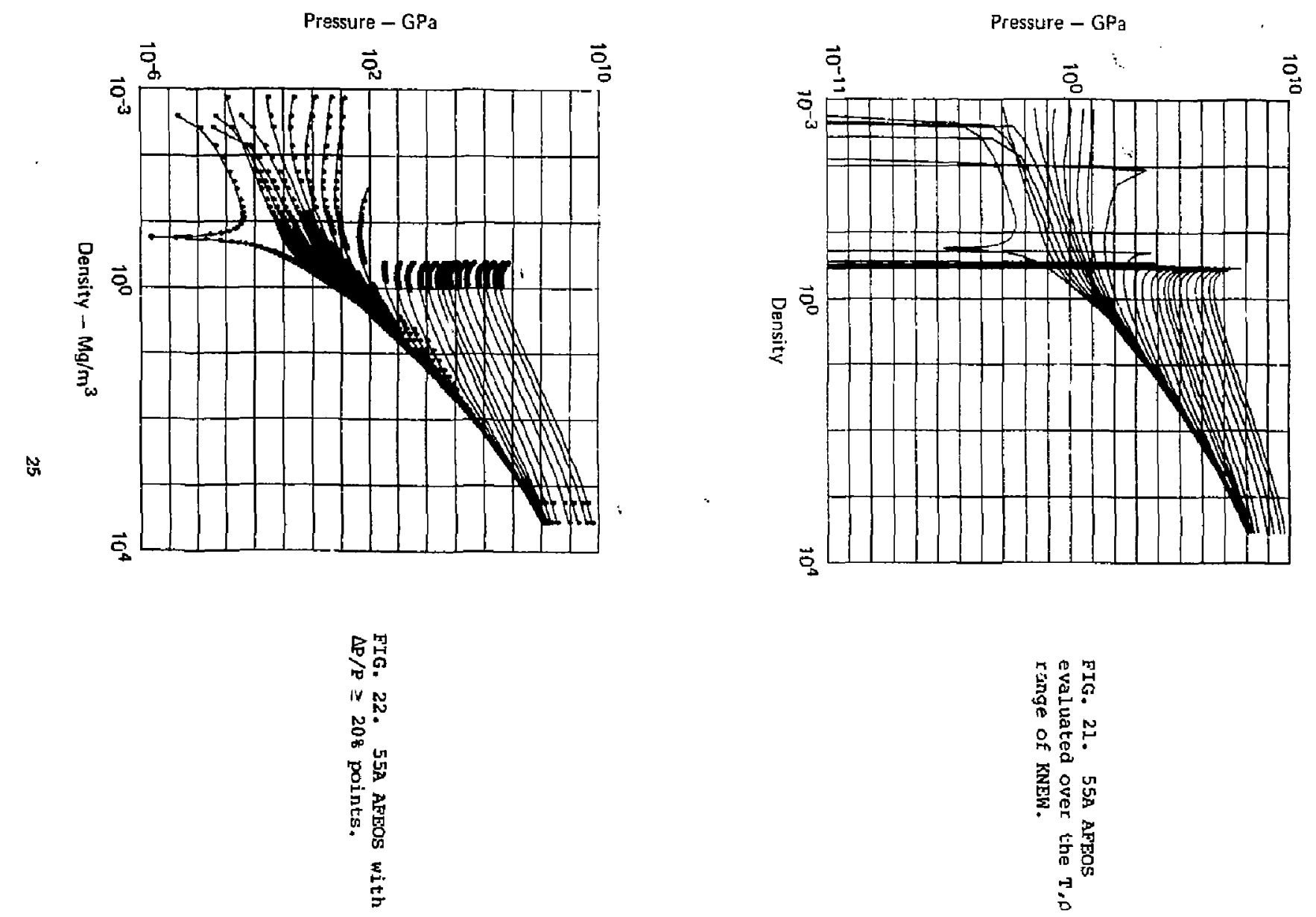

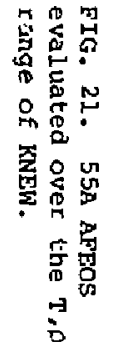



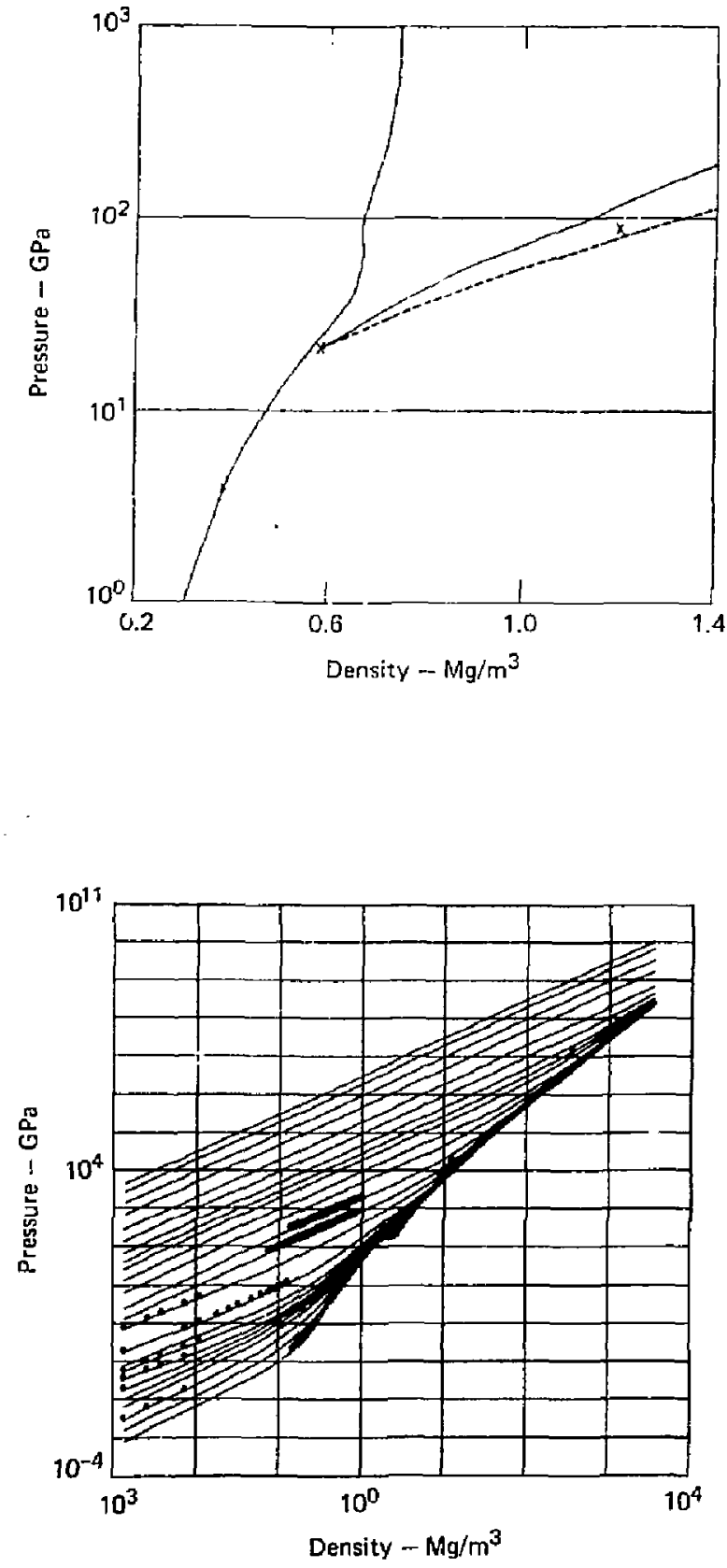

EIG. 23. Shock loci flom 55A AFEOS and the KNEW PDB, compared to shock data. Nate that the poor quality analytic fit comes mich closer to the B70-kbar experimental point due to weighting.
FIG. 24. 55B AFEOS evaluated over the $T, \rho$ range of INEW. $\triangle \mathrm{P} / \mathrm{P} \geq 20$ \& erroi points superimposed, showing a high quality fit to the underlying data. 

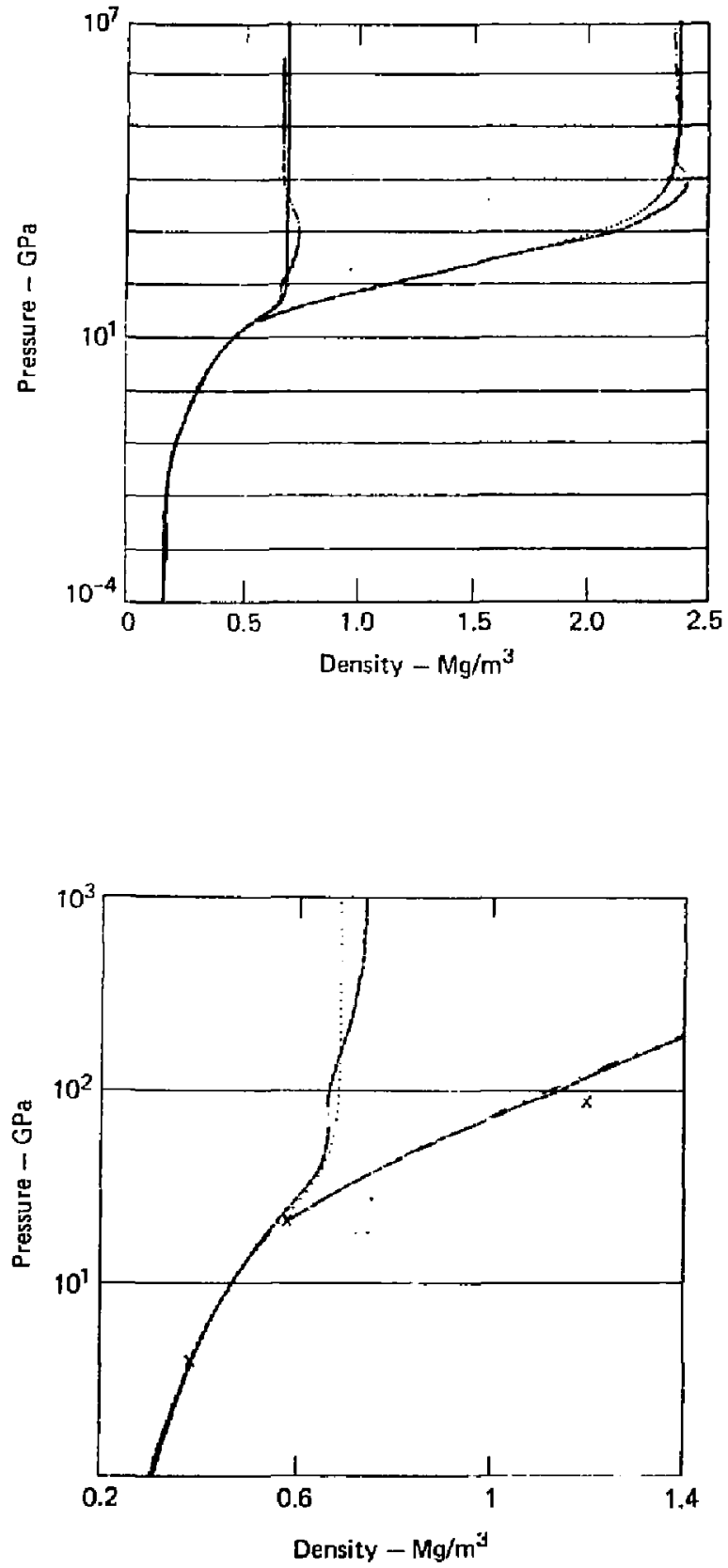

27
FIG. 25. Principal Hugoniot and ceflected shork Locus from AF55B compared to the same tracks computed frOm KNEW PDB.
FIG. 26. Shock loci from AF55B and KNEW, sompared to shocik data. 

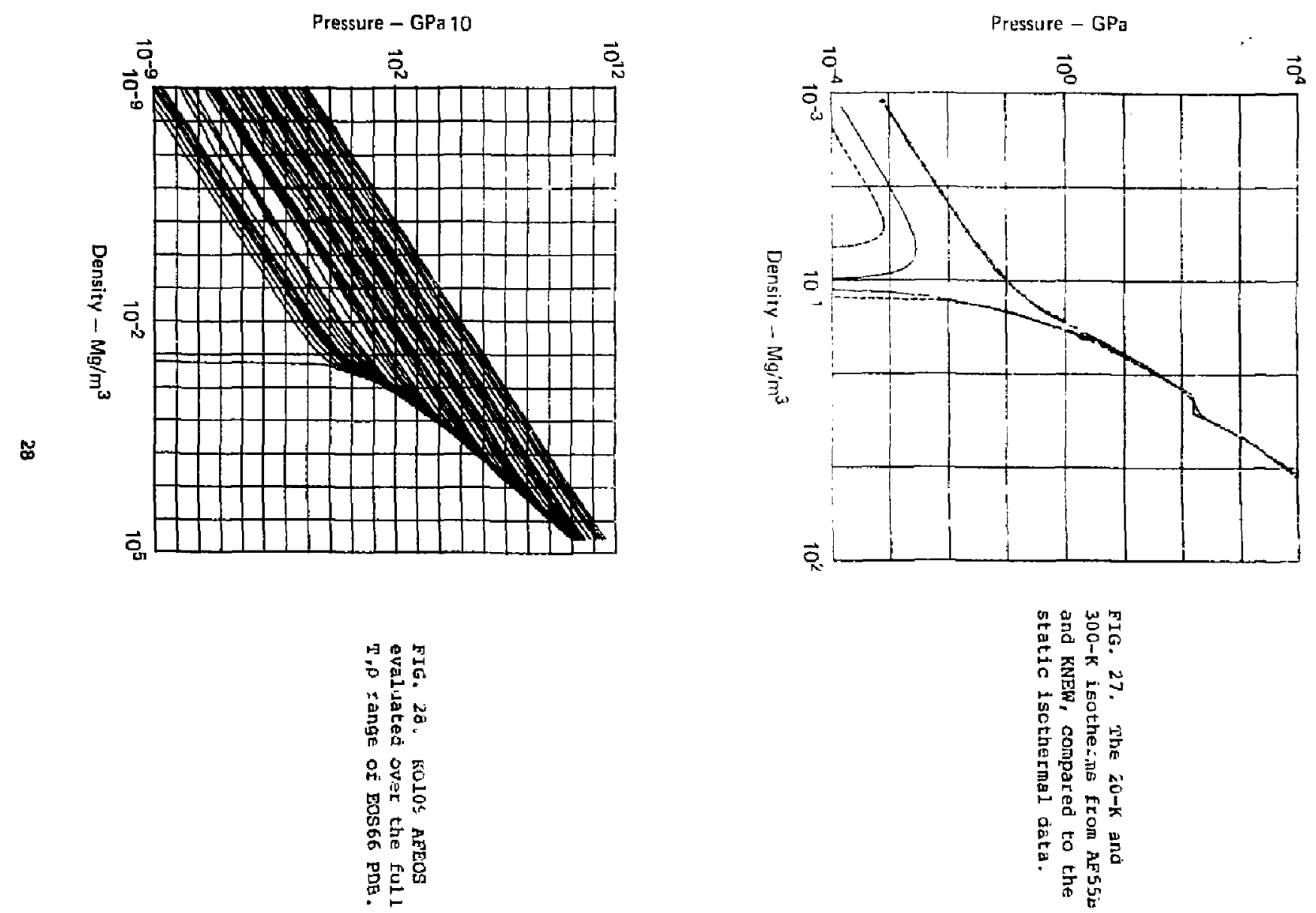

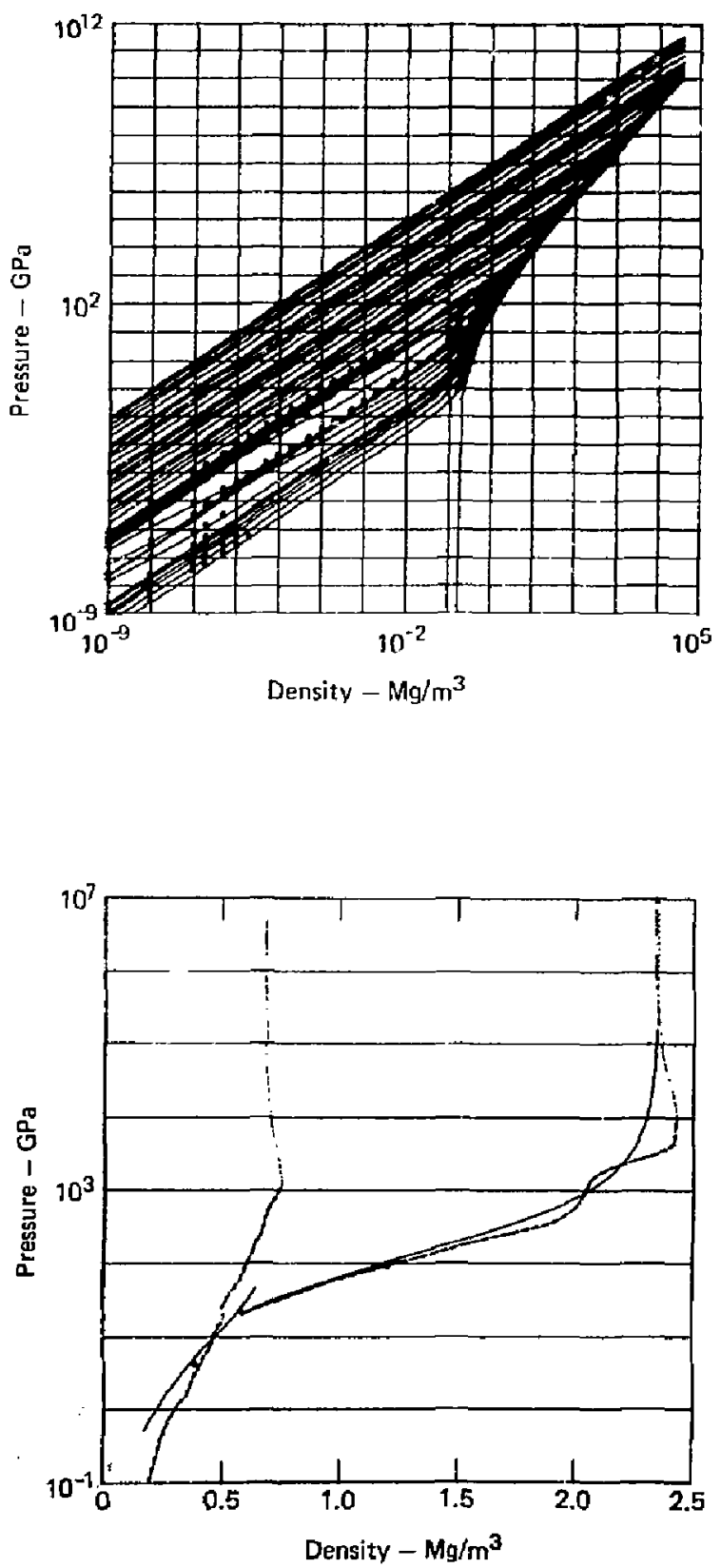

FIG. 29. KO109 AFEOS with $\triangle P / P \geq 208$ error points are superimposed.
FIG. 30. Principal Hugoniot and double-shock locus from KOLO9 and EOS66 compared. 

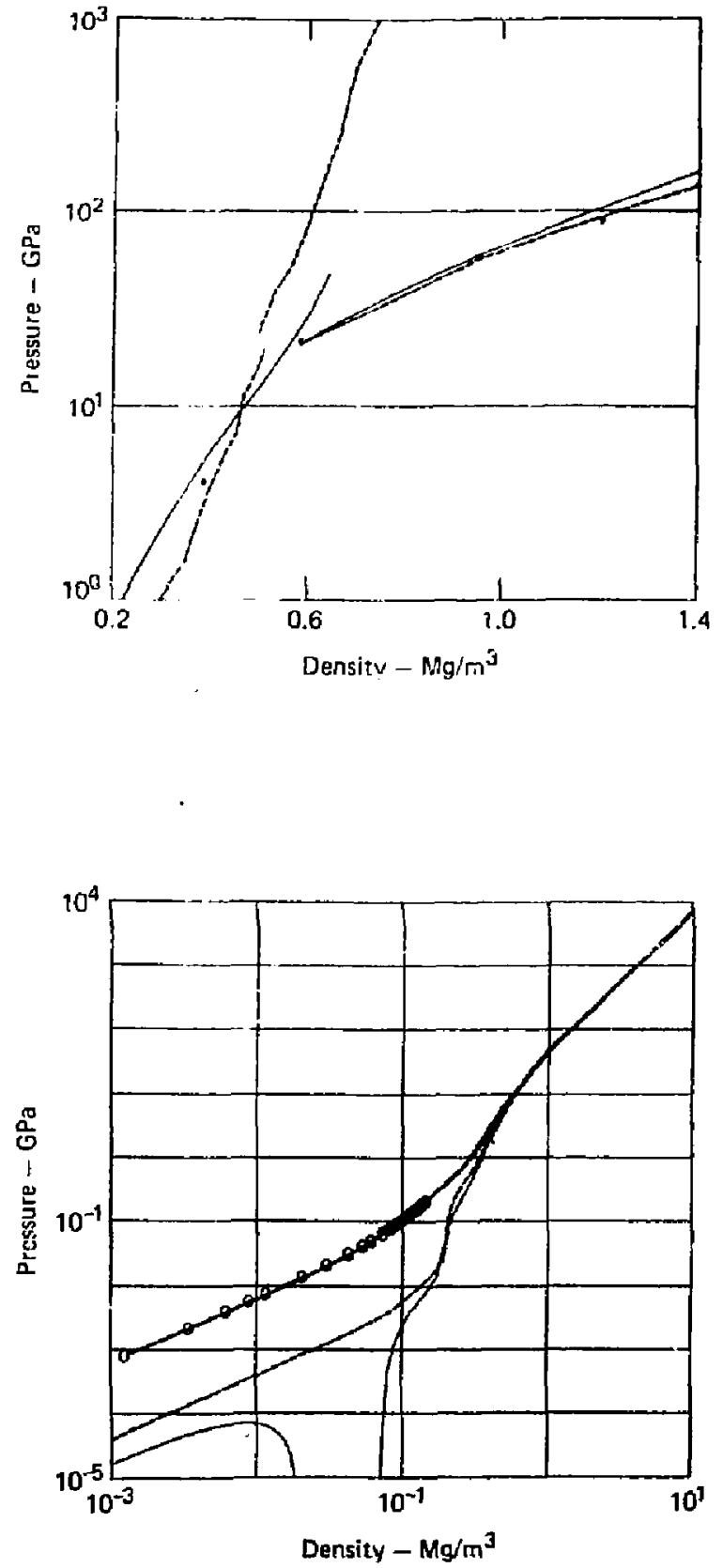

EIG, 31. Shock loct fron Rolog and Eos66 compared with experimental shockwàve data.
FIG. 32. The 20-K and 3DD-K isotherms from Kol09 and EOS66 compared to the experimental data. 

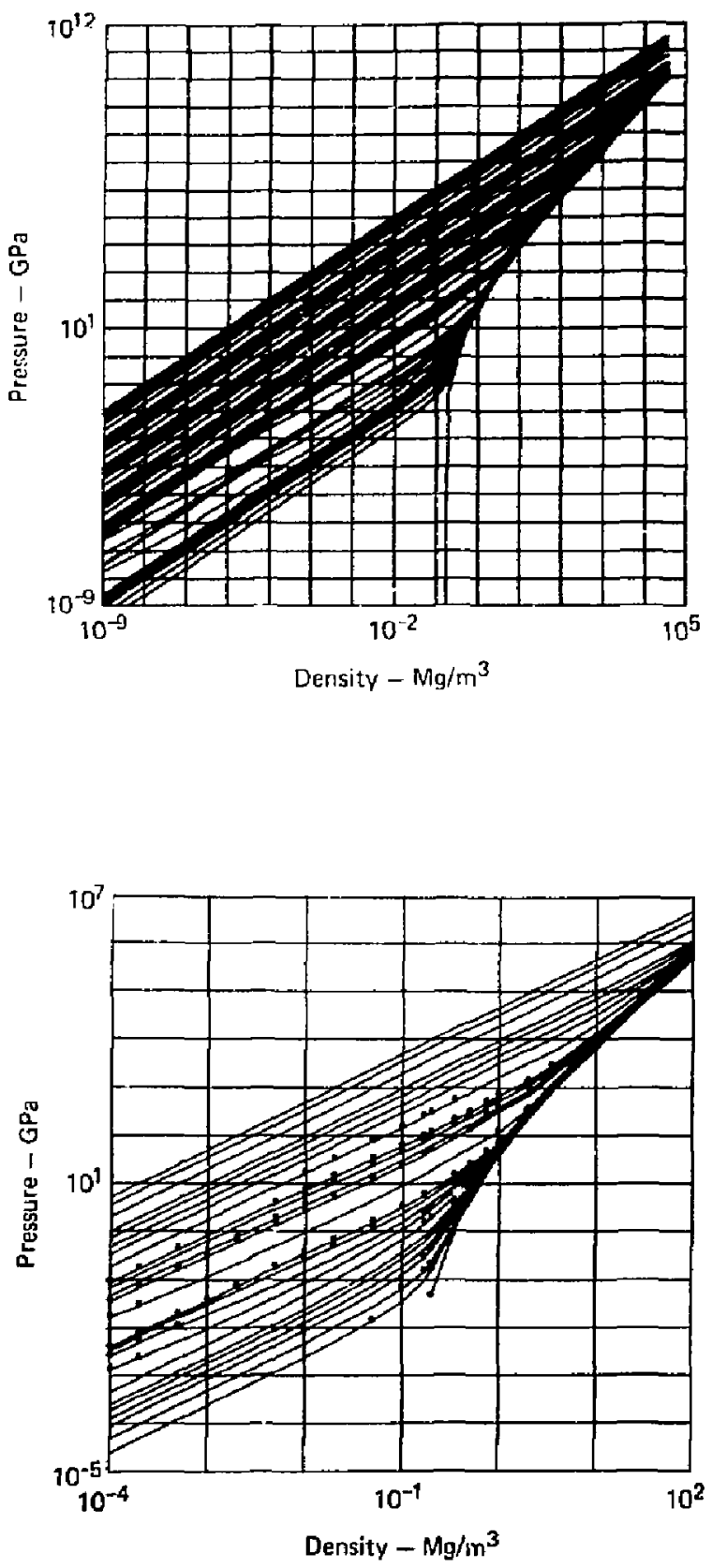

FIG. 33. AFY5A pressure surface evaluated over the full T,p range of Eos95.
FIG. 34. Segment of same surface with $\Delta P / P \geq 10 \%$ ercor points superimposed. 

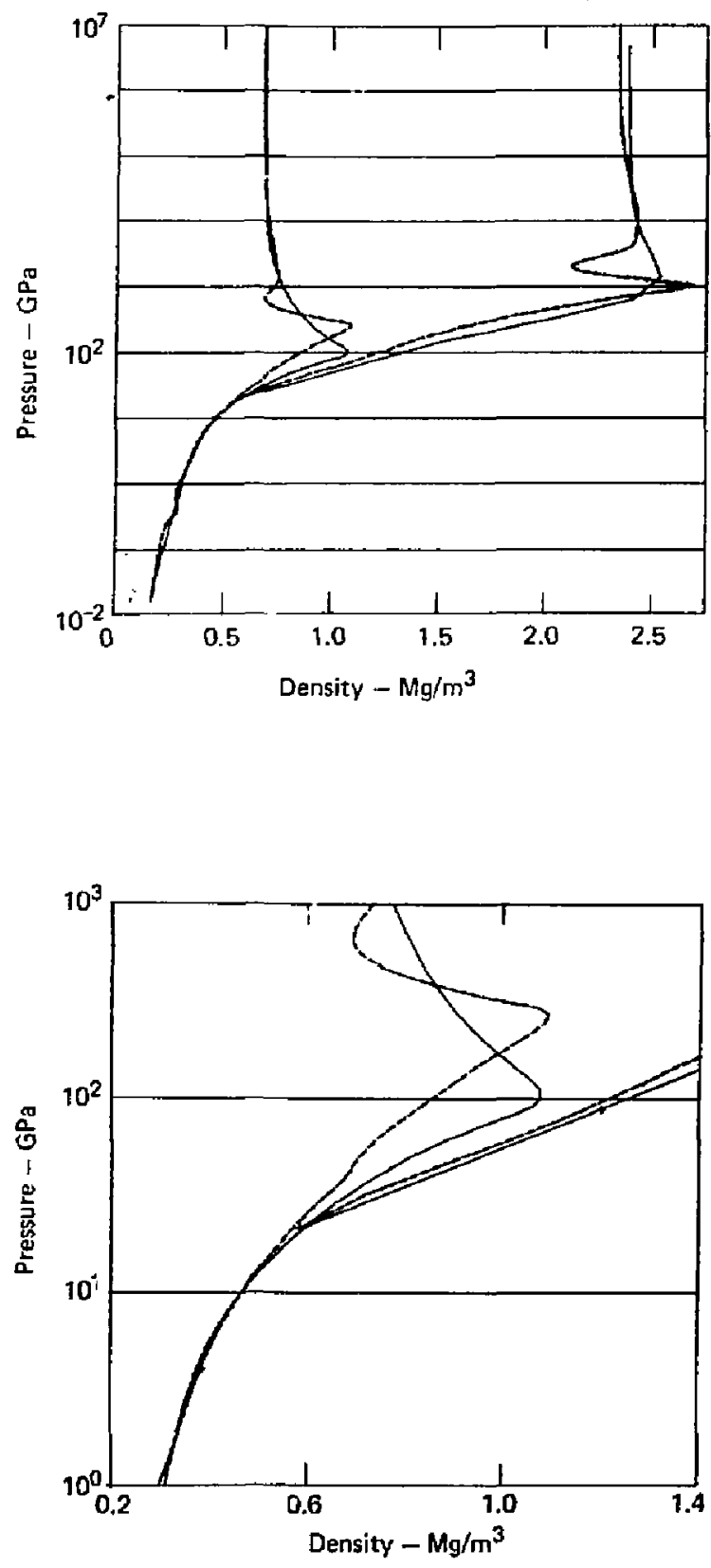

FIG. 35. Principal hugoniot and reflected-shock locus calculated from AF95A and the EOS95 RDB. Even thc very high quality $A F$ does not give $P(\rho, E)$ surfaces which track the PDB Hugoniot closely at all points. Weighting the AF to the experimental data may be responsible.
FIG. 36. The two shock loci from AF95A and EOS95, compired to the experimental shock data. 


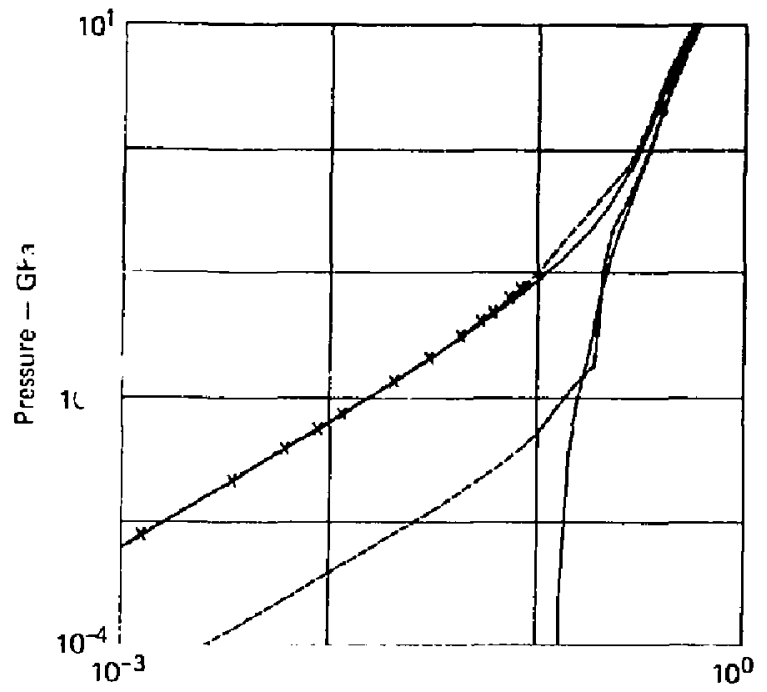

Density $-\mathrm{Mg} / \mathrm{m}^{3}$

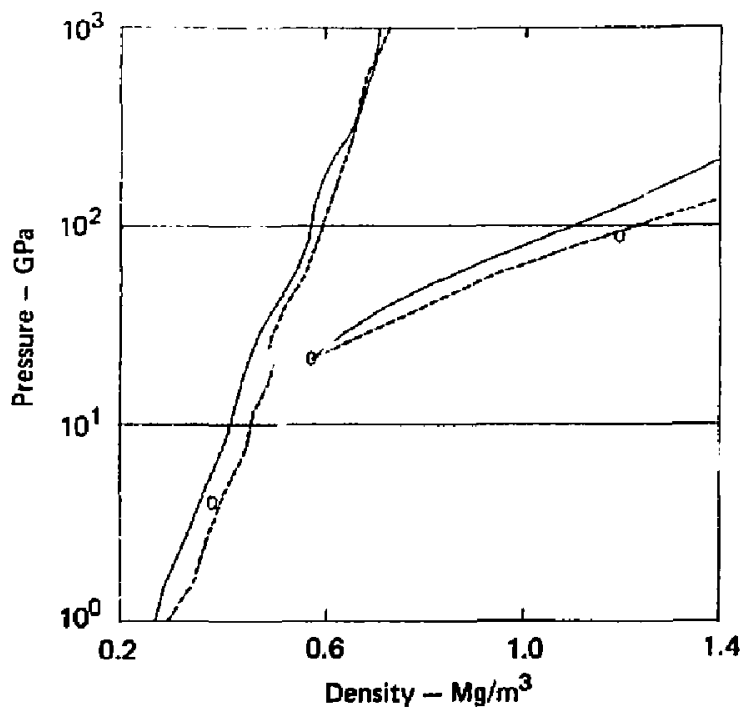

FIG. 37. The 20-K and $300-\mathrm{K}$ isotherms from AF95A and EOS95, compared to the static isothermal data.
FIG. 38. Principal Hugoniot and double-shock locus from BoL275 (trace) and EOS66 (dash), compared to the experimental data. 

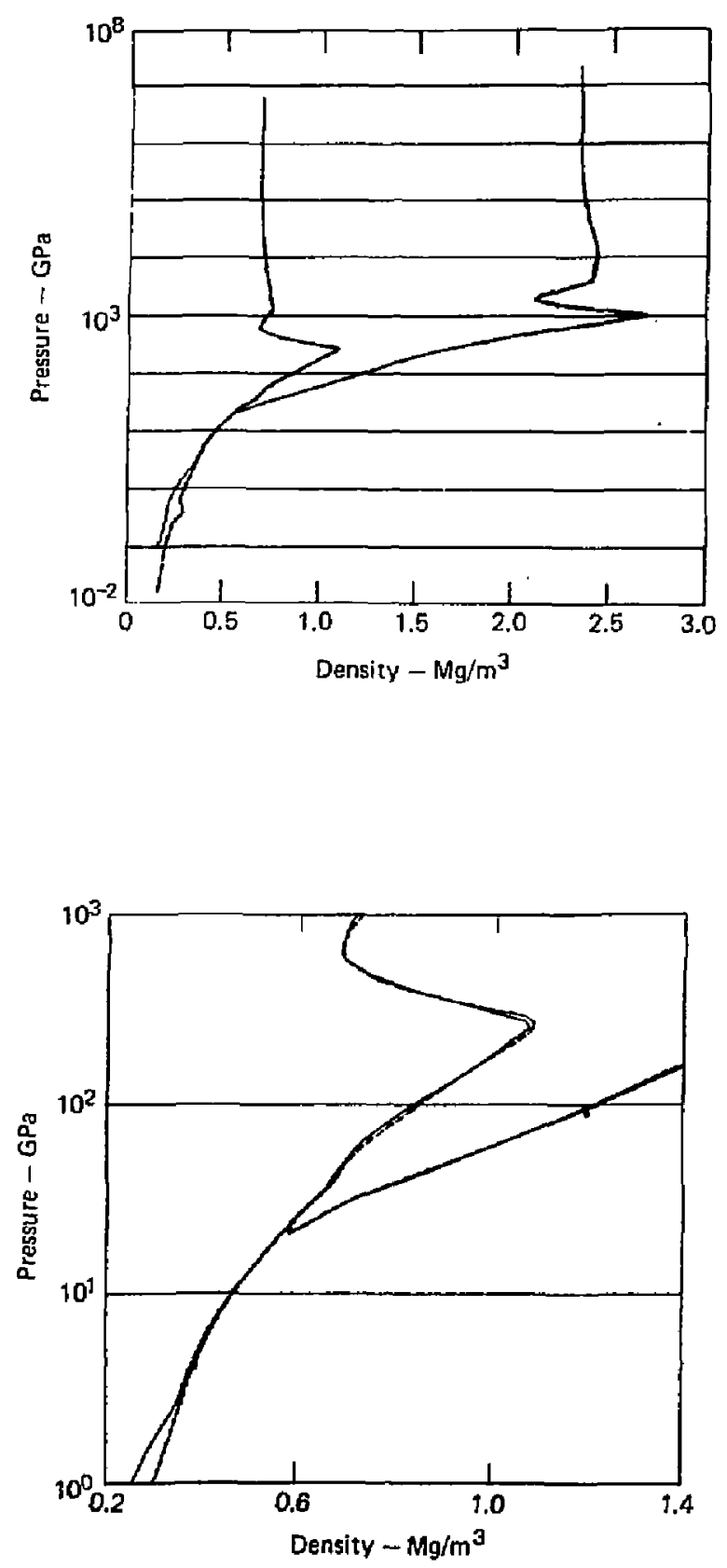

FIG. 39. Principa1 Hugoniot and double-shock locus From $\mathrm{BQL} 277$ and EOS95.
FIG, 40. Two strock loci from BQL277 and EOS95, compared to the experimental data. 


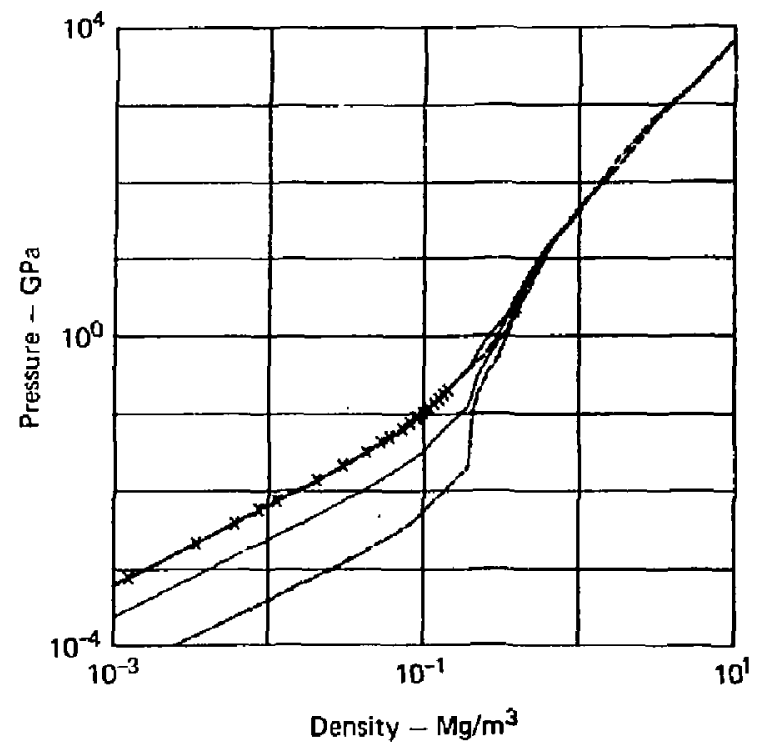

FIG. 41. The 20-K and 300- $K$ isotherms fIon BQL277 and E0595, compared to the experimental data.

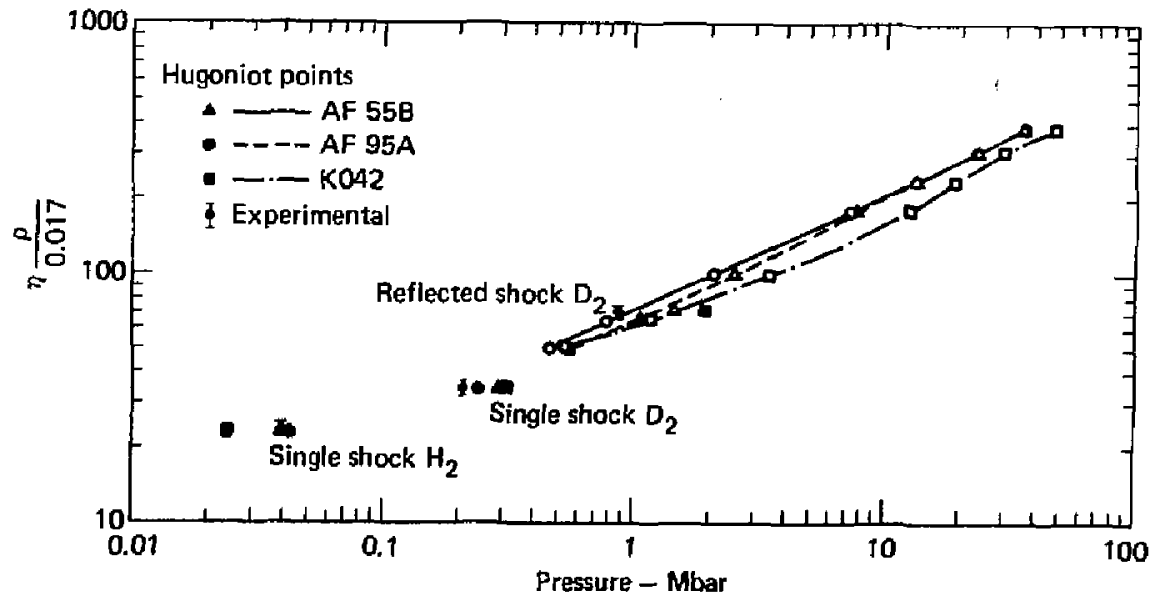

FIG. 42. Carlson' $\mathrm{E}, \mathrm{n}$ sequence, as evaluated by different EOS's, shown as curves. The Hugoniot points for the single- and double-shock points for the different EOS's are al:o shown with the experinental data. 
1. R. J. Olness, H. C. Graboske, and W. G. Hoover, New Equation of State for Hydrogen, Lawrence Livermore Laboratory, Livermore, CA, Rept. UCID-16095 (1972).

2. H. C. Graboske, R. J. O1ness, and A. S. Grossman, "Thermodynamies of Dense Hydrogen-Helium," in Astrophys. J. 199, 255 (1975).

3. G. Kerley, "Equation of State and Phase Diagram of Dense Hydrogen," in Phys. Earth Plan. Int, 6,78 (1972).

4. A. S. Grossman, J. B. Pcllack, R. T. Reynolds, A. L. Summers, and H. C. Graboske, "The Effect of Dense Cores on the Structure and Evolution of Jupiter and Saturn," Icarus (to be published).

5. H. C. Graboske and R. J. Olness, Thermodynamic Consistency in Theoretical Equatior, of State, Lawrence Livermore Laboratory, Livermore, CA, Rept. UCIR-811 (1974).

6. J. W. Stewart, "Compression of Solidified Gases to $20,000 \mathrm{~kg} / \mathrm{cm}^{2}$ at Low Temperatures," in J. Phys. Chem. Solids 1 . 146 (1956).

7. M. van Thiel and B. J. Alder, "Shock Compression of Liquid Hydrogen," in Molecular Phys. 19, 427 (1966).

8. M. van Thiel, L. B. Lord, H. H. Gust, A. C. Mitchell, and M. D'Addario (all of Lawrence Livermore Laboratory) and $\mathrm{K}$. Boutwell, E. Wilbarger, and B. Barrett (all of Delco Electronics Division, G.M. Corp., Goleta, CA), "Shock Compressicn of Deuterium to $900 \mathrm{kbar}, "$ in phys. Earth plan. Int. 9. 57 (1974).

9. R. Dick, unpublished, LOS Alamos Scientific Laboratory, LOS Alamos, NM (1972).

10. M. Ross and $c$. Shishkevish, Molecular and Metallic Hydrogen, Rand Corp., Sante Monica, CA, Rept. R-2056-ARPA (1977), and M. Ross, Lawrence Livermore Laboratory, Livermore, CA, private communication (1979).

11. H. Graboske, H Division Equation of State Reference File (HERF) Index. Lawrence Livernore Laboratory, Livermore, CA (1979).

12. See L. Marino (Lawrence IIvermore Laboratory, Iivermore, CA) for details of his comparison. 